\title{
SECOND-PRICE ALL-PAY AUCTIONS AND BEST-REPLY MATCHING EQUILIBRIA
}

\author{
Gisèle UMBHAUER* \\ *Bureau d'Economie Théorique et Appliquée, University of Strasbourg, \\ Strasbourg, France \\ umbhauer@unistra.fr \\ Accepted October 2018
}

Published in International Game Theory Review, Vol 21, Issue 2, 40 pages, 2019

DOI 10.1142/S0219198919400097

\begin{abstract}
The paper studies second-price all-pay auctions - wars of attrition - in a new way, based on classroom experiments and Kosfeld et al.'s best-reply matching equilibrium. Two players fight over a prize of value $\mathrm{V}$, and submit bids not exceeding a budget $\mathbf{M}$; both pay the lowest bid and the prize goes to the highest bidder. The behavior probability distributions in the classroom experiments are strikingly different from the mixed Nash equilibrium. They fit with best-reply matching and generalized bestreply matching, an ordinal logic according to which, if bid $\mathrm{A}$ is the best response to bid $\mathrm{B}$, then $\mathrm{A}$ is played as often as B. The paper goes into the generalized best-reply matching logic, highlights the role of focal values and discusses the high or low payoffs this logic can lead to.

Keywords : second-price all-pay auction; war of attrition; best-reply matching; Nash equilibrium; classroom experiment.
\end{abstract}

Subject Classification: C72, D44.

\section{Introduction}

The paper studies second-price all-pay auctions - wars of attrition - in a new way, based on classroom experiments and Kosfeld et al.'s [2002] best-reply matching equilibrium. A lot has been said about first-price all-pay auctions, but there are only few papers with experiments on second-price all-pay auctions (see Hörisch and Kirchkamp [2010] and Dechenaux et al. [2015] for experiments with this class of games). The second-price allpay auction studied in this paper is the most standard one: two players fight over a prize of value $\mathrm{V}$, have a budget $\mathrm{M}$, and simultaneously submit bids not exceeding $\mathrm{M}$. Both pay the lowest bid and the prize goes to the highest bidder; in case of a tie, each player gets the prize with probability $1 / 2$.

The mixed Nash equilibrium is strikingly different from the behavior observed in the classroom experiments (an experiment with 116 students, another with 109 students). The distributions are so different that we cannot conclude on overbidding or underbidding in comparison with the Nash equilibrium behavior. This observation is partly shared by Hörisch and Kirchkamp [2010]: whereas overbidding is regularly observed in first-price all-pay auction experiments (see for example Gneezy and Smorodinsky [2006] and 
Lugovskyy et al. [2010]), Hörisch and Kirchkamp [2010] establish that underbidding prevails in sequential war of attrition experiments.

In our classroom experiments, the students' behavior better fits with best-reply (and generalized best-reply) matching, a behavioral concept developed by Kosfeld et al. [2002]. According to best-reply matching, if bid A is the best response to bid $\mathrm{B}$, then A is played as often as B. Mixed Nash equilibria and best-reply matching equilibria follow a different logic: whereas Nash equilibrium probabilities are calculated so as to equalize the payoffs of the bids played at equilibrium, best-reply matching probabilities just aim to match best responses, each bid being played as often as the bid to which it is a best reply.

In the second-price all-pay auction, there are multiple best responses to a bid, when the bid does not exceed the value of the prize. In case of multiple best responses, best-reply matching requires that each best response be played with the same probability, whereas generalized best-reply matching (Umbhauer [2016]) allows any probability distribution over the set of best responses. So we mostly work with generalized best-reply matching, given that real players may spontaneously select some best responses more than others. We say that some best responses are more focal than others and we exploit this characteristic to come closer to the students' behavior.

We also call attention to the payoffs of the bidders. In the mixed Nash equilibrium, the payoffs are slightly positive in the discrete setting (they are null in a continuous setting). This is no longer true with best-reply and generalized best-reply matching: the players can lose or win a lot of money.

Section 2 recalls the pure and mixed Nash equilibria in the discrete second-price all-pay auctions, when $\mathrm{M}, \mathrm{V}$ and the bids are integers. It also presents the two classroom experiments and compares the students' behavior with the mixed Nash equilibrium. In section 3 , we present the best-reply matching equilibrium and the generalized best-reply matching equilibrium, and we compare the mixed Nash equilibrium philosophy with the best-reply matching philosophy. In section 4 we establish the best-reply matching equilibria and compare them to the students' behavior. Section 5 is the main part. We turn to generalized best-reply matching and focal behavior. Given that there are multiple best responses to a given bid, players may only play some focal best responses. Focusing on the best responses $0, \mathrm{~V}$ and $\mathrm{M}$ leads to a generalized best-reply matching equilibrium which is close to the students' behavior. And focusing on cautious best responses leads to a generalized best-reply matching equilibrium that is also, surprisingly, a generalized bestreply matching equilibrium of the first-price all-pay auction. Section 6 is on the mixed Nash equilibrium payoffs and the (generalized) best-reply matching equilibrium payoffs. Section 7 concludes on the role of $\mathrm{M}$ in second-price all-pay auctions.

\section{Classroom Experiments, Nash Equilibria and Students' Behavior}

Two players have a budget M. They fight over a prize of value V. Each player i submits a bid $\mathrm{b}_{\mathrm{i}}, i=1,2$ lower than or equal to $\mathrm{M}$. The prize goes to the highest bidder but both bidders 
pay the lowest bid. In case of a tie, the prize goes to each bidder with probability $1 / 2$. Throughout the paper, we suppose $M \geq V . \mathrm{V}, \mathrm{M}$ and the rules of the game are common knowledge. Moreover, in the classroom experiments, $\mathrm{M}, \mathrm{V}$ and the bids are integers. So we restrict attention to discrete games.

This game is known to have a lot of intuitive asymmetric Nash equilibria, one player bidding nothing ( 0 ), the other bidding $\mathrm{V}$ or more. In some way, if the first player is cautious and afraid of losing money, whereas the second player is a hothead, the first player bids 0 , whereas the second can afford to bid M (even if M is much larger than V), getting the prize without paying anything, thanks to the cautious behavior of the first player. Second-price all-pay auctions are rightly seen as dangerous games with great opportunities: when a hothead meets another one, both lose a lot of money, but if he meets a cautious player, then he wins $\mathrm{V}$ and pays nothing. And if a cautious player meets another one, each player gets the expected amount $\mathrm{V} / 2$ without paying anything.

Things become less intuitive when turning to the unique mixed Nash equilibrium (NE) of this game, which is a symmetric mixed NE. We call $\mathrm{q}_{\mathrm{i}}$ the probability of bidding $\mathrm{i}$.

Result 1 Umbhauer [2017] ${ }^{\mathrm{a}} . V, M$ and the bids are integers, $M \geq V$ and the bids go from $O$ to $M$; we assume that $V$ is odd. The unique mixed $N E$ is symmetric.

We note $\bar{\imath}=M-V / 2-5 / 2$. The main recurrence equation that defines the probabilities is: $q_{i=} 2 q_{i+1} / V+q_{i+2} \quad$ ifrom 0 to $\bar{\imath} \quad($ for $\bar{\imath} \geq 0)$.

The additional equations are:

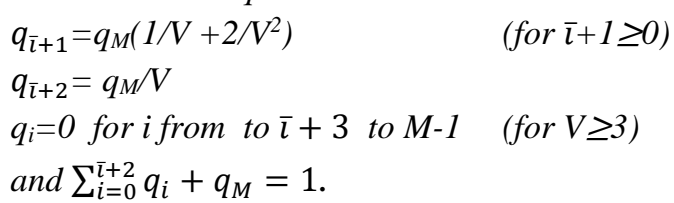

Proof see Appendix A.

We study the mixed $\mathrm{NE}$ for $(\mathrm{V}=3, \mathrm{M}=5),(\mathrm{V}=3, \mathrm{M}=6)$ (these equilibria are necessary in the classroom experiments) and ( $\mathrm{V}=9, \mathrm{M}=12)$. The $\mathrm{NE}$ are given in Table 1 and illustrated in Fig. $1 \mathrm{a}, 1 \mathrm{~b}$ and $1 \mathrm{c}$.

The shape of the three distributions is close to that obtained in the continuous setting, illustrated in Fig. 1b (the decreasing curve, the segment and the point) and briefly recalled below (Result 2). This similarity is always observed when $\mathrm{V}, \mathrm{M}$ and the bids are integers and $\mathrm{V}$ is odd (see Umbhauer [2017]).

\footnotetext{
${ }^{\text {a }}$ Result 1 only holds for odd values of $\mathrm{V}$ ( $\mathrm{V}$ and $\mathrm{M}$ being integers and the bids being all integers from 0 to $\mathrm{M}$ ). When $\mathrm{V}$ is even ( $\mathrm{V}$ and $\mathrm{M}$ being integers, the bids being all integers from 0 to $\mathrm{M}$ ), a different result applies, due to a different gap between $\mathrm{M}-\mathrm{V} / 2$ and the highest played bid lower than $\mathrm{M}$ in case of $\mathrm{V}$ odd and $\mathrm{V}$ even. This has a strong impact on the probability distribution, that is much less regular in shape for $\mathrm{V}$ even than for $\mathrm{V}$ odd (see Umbhauer [2017]).
} 
Table 1. Mixed Nash equilibria for ( $V=3, M=5),(V=3, M=6),(V=9, M=12)$.

\begin{tabular}{|c|c|c|c|c|c|c|c|c|c|c|}
\hline & $\mathrm{q}_{0}$ & $\mathrm{q}_{1}$ & $\mathrm{q}_{2}$ & $\mathrm{q}_{3}$ & $\mathrm{q}_{4}$ & q5 & q6 & $\mathrm{q}_{7}$ & $\begin{array}{l}\mathrm{q}_{8}, \mathrm{q}_{9}, \\
\mathrm{q}_{10}, \mathrm{q}_{11}\end{array}$ & $\mathrm{q}_{12}$ \\
\hline $\begin{array}{l}V=3 \\
M=5\end{array}$ & $\begin{array}{l}83 / \\
293= \\
0.283\end{array}$ & $\begin{array}{l}57 / \\
293= \\
0.195\end{array}$ & $\begin{array}{l}45 / \\
293= \\
0.154\end{array}$ & $\begin{array}{l}27 / \\
293= \\
0.092\end{array}$ & 0 & $\begin{array}{l}81 / \\
293= \\
0.276\end{array}$ & & & & \\
\hline $\begin{array}{l}V=3 \\
M=6\end{array}$ & $\begin{array}{l}337 / \\
1216= \\
0.277\end{array}$ & $\begin{array}{l}249 / \\
1216= \\
0.205\end{array}$ & $\begin{array}{l}171 / \\
1216= \\
0.141\end{array}$ & $\begin{array}{l}135 / \\
1216= \\
0.111\end{array}$ & $\begin{array}{l}81 / \\
1216= \\
0.066\end{array}$ & 0 & $\begin{array}{l}243 / \\
1216= \\
0.20\end{array}$ & & & \\
\hline $\begin{array}{l}\mathrm{V}=9 \\
\mathrm{M}=12\end{array}$ & 0.106 & 0.093 & 0.085 & 0.074 & 0.069 & 0.058 & 0.056 & 0.046 & 0 & 0.413 \\
\hline
\end{tabular}

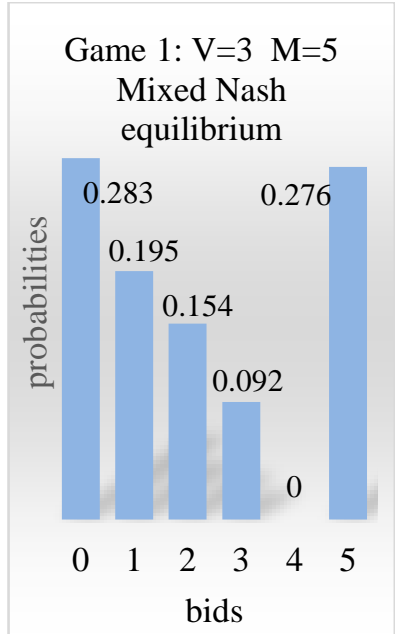

Fig. 1a. Mixed NE ( $V=3, M=5)$.

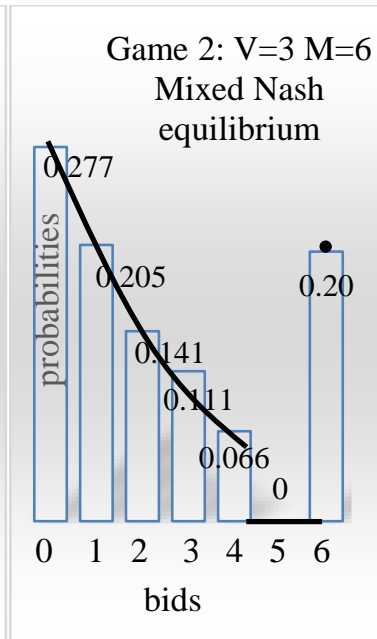

Fig. 1b. Mixed NE $(V=3, M=6)$

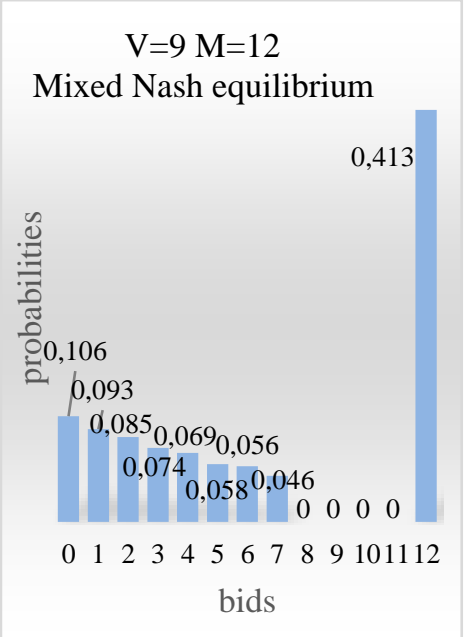

Fig. 1c. Mixed NE (V=9,M=12).

Result 2. We call $b$ a bid in $[0, M]$. The unique mixed NE in the continuous game is symmetric and given by:

- $\quad$ The support of the equilibrium is $[0, M-V / 2] \cup\{M\}$,

- the cumulative probability distribution on $[0, M-V / 2]$ is given by $F(b)=1-e^{-b / V}$,

- $\quad M$ is a mass point played with probability $\quad l-F(M-V / 2)=e^{I / 2-M / V}$.

The net payoff (payoff minus $M$ ) is equal to 0 at equilibrium.

Proof see Appendix B.

It derives from the Fig. 1a, $1 \mathrm{~b}$, and $1 \mathrm{c}$ that the NE probabilities decrease from 0 to $\mathrm{M}-\mathrm{V} / 2$ $1 / 2$ (M-V/2 in the continuous setting), and are null from $\mathrm{M}-\mathrm{V} / 2+1 / 2$ to $\mathrm{M}-1$ (in $] \mathrm{M}-\mathrm{V} / 2$, $\mathrm{M}$ [ in the continuous setting), whereas bid $\mathrm{M}$ is played with a positive probability. In the continuous setting, $\mathrm{M}$ is a mass point; this is rather intuitive, in that $\mathrm{M}$ is a focal point with a special property. Given that nobody can play more than $\mathrm{M}$ and given that many players bid less, a player, when he bids $M$, is sure to get the prize with a high probability and he often pays less than $\mathrm{V}$, especially if $M-V / 2<V$. In this latter case, at equilibrium, bidding $\mathrm{M}$ leads to a negative payoff only if the opponent bids $\mathrm{M}$ too. 
0 is more often played than the bids from 1 to $\mathrm{M}-\mathrm{V} / 2-1 / 2$, which is rather intuitive, because 0 is the only best reply to $\mathrm{M}$ and never leads to losing money. Yet the probability of playing 0 seems unrelated to the probability of playing $M\left(q_{0}\right.$ is very close to, larger than, much smaller than $\mathrm{q}_{\mathrm{M}}$, respectively in Fig. 1a, 1b and 1c) and this non-intuitive fact is confirmed in the continuous setting: 0 is not a mass point and the cumulative probability on $[0, \mathrm{db}]$ is $\mathrm{db} / \mathrm{V}(d b \rightarrow 0)$ so does not depend on $\mathrm{M}$, in contrast to the probability assigned to $\mathrm{M}$. It namely follows that when $\mathrm{V}$ and $\mathrm{M}$ become large, but $\mathrm{M} / \mathrm{V}$ remains constant, the cumulative probability on $[0, \mathrm{db}]$ tends towards 0 whereas $\mathrm{f}(\mathrm{M})$ remains constant. This is not intuitive.

What about risk aversion? In the above equilibria, we implicitly suppose that the players are risk neutral, given that the utility is assumed to be equal to the payoff. We could follow Hörisch and Kirchkamp [2010], and opt for the utility function $U(x)=e^{-r M}-e^{-r x}$ to express risk aversion ( $r$ being the player's degree of risk aversion). This would lead to steeper density probability distributions, with a stronger probability on low bids, but it would not change the nature of the mixed NE.

We now present the two classroom experiments.

In the first classroom experiment ${ }^{\mathrm{b}}, 116$ L3 students, i.e. undergraduate students in their third year of training, played the second-price all-pay auction game in matrix 1 (Game 1), with $\mathrm{V}=3, \mathrm{M}=5$ and the possible bids $0,1,2,3,4$ and 5 . In the second classroom experiment, $109 \mathrm{~L} 3$ students played the second-price all-pay auction game in matrix 2 (Game 2), with $\mathrm{V}=30, \mathrm{M}=60$ and the possible bids $0,10,20,30,40,50$ and 60 . This second game has the same best responses and the same $\mathrm{NE}$ as the game with $\mathrm{V}=3, \mathrm{M}=6$, and bids $0,1,2,3,4,5$ and 6 (to get the payoffs in the game with $V=3, M=6$, it is sufficient to divide the payoffs of Game 2 by 10). So, to get the mixed NE of Game 2, we can apply result 1 to the game with $\mathrm{V}=3, \mathrm{M}=6$ and bids $0,1,2,3,4,5,6$.

\section{Player 2}

\begin{tabular}{|c|c|c|c|c|c|c|c|}
\hline & & 0 & 1 & 2 & 3 & 4 & 5 \\
\hline \multirow{6}{*}{ Player 1} & 0 & $(6.5,6.5)$ & $(5,8)$ & $(5,8)$ & $(5,8)$ & $(5,8)$ & $(5,8)$ \\
\hline & 1 & $(8,5)$ & $(5.5,5.5)$ & $(4,7)$ & $(4,7)$ & $(4,7)$ & $(4,7)$ \\
\hline & 2 & $(8,5)$ & $(7,4)$ & $(4.5,4.5)$ & $(3,6)$ & $(3,6)$ & $(3,6)$ \\
\hline & 3 & $(8,5)$ & $(7,4)$ & $(6,3)$ & $(3.5,3.5)$ & $(2,5)$ & $(2,5)$ \\
\hline & 4 & $(8,5)$ & $(7,4)$ & $(6,3)$ & $(5,2)$ & $(2.5,2.5)$ & $(1,4)$ \\
\hline & 5 & $(8,5)$ & $(7,4)$ & $(6,3)$ & $(5,2)$ & $(4,1)$ & $(1.5,1.5)$ \\
\hline
\end{tabular}

Matrix $1:$ Game $1, \mathrm{~V}=3, \mathrm{M}=5$.

\footnotetext{
b This experiment has also been partly studied in Umbhauer [2016].

${ }^{c}$ For reasons of presentation homogeneity, it would be logical to give the students' results by referring to the game with $\mathrm{V}=3, \mathrm{M}=6$ and the bids $0,1,2,3,4,5$ and 6 . But of course this cannot be done from an experimental point of view.
} 
Player 2

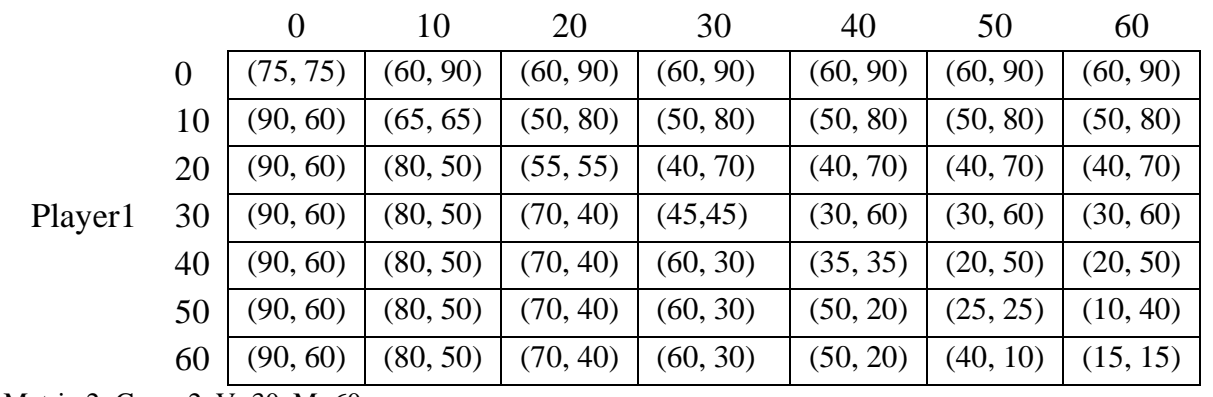

Matrix 2: Game 2, V=30, $M=60$.

The games were played during game theory lectures and the students knew what a normalform game is. So they had no difficulty in understanding the two games, and the meaning of the normal forms in matrix 1 and matrix 2. Several couples of payoffs were explained in detail to the students, to ensure that they understood the rules of the game. The students had the matrices, as well as the explanation of the couples of payoffs, in front of them while choosing their bid. We add that the first game was played by students trained in NE and dominance. In contrast, the second game was played by novice students with no training in NE and dominance. Students were also allowed, but not compelled to, add comments on their way of playing.

The students' way of playing is given in Table 2 .

Table 2. Comparison of the mixed NE and the students' behavior.

\begin{tabular}{cccccc}
\hline $\begin{array}{c}\text { Game1 } \\
\text { V=3, } \\
\mathbf{M = 5} \\
\text { Bids }\end{array}$ & $\begin{array}{l}\text { Nash } \\
\text { equilibrium } \\
\text { probabilities } \\
\text { (percentage) }\end{array}$ & $\begin{array}{l}\text { Students: } \\
\text { frequencies } \\
\text { of the bids }\end{array}$ & $\begin{array}{c}\text { Game2 } \\
\text { V=30, } \\
\text { M=60 } \\
\text { bids }\end{array}$ & $\begin{array}{l}\text { Nash } \\
\text { equilibrium } \\
\text { probabilities } \\
\text { (percentage) }\end{array}$ & $\begin{array}{l}\text { Students: } \\
\text { frequencies } \\
\text { of the bids }\end{array}$ \\
\hline $\mathbf{0}$ & $28.3 \%$ & $37.9 \%$ & 0 & $27.7 \%$ & $33 \%$ \\
\hline $\mathbf{1}$ & $19.5 \%$ & $9.5 \%$ & 10 & $20.48 \%$ & $5.5 \%$ \\
\hline $\mathbf{2}$ & $15.4 \%$ & $1.7 \%$ & 20 & $14.06 \%$ & $2.8 \%$ \\
\hline $\mathbf{3}$ & $9.2 \%$ & $20.7 \%$ & 30 & $11.1 \%$ & $21.1 \%$ \\
\hline $\mathbf{4}$ & 0 & $15.5 \%$ & 40 & $6.66 \%$ & $4.6 \%$ \\
\hline $\mathbf{5}$ & $27.6 \%$ & $14.7 \%$ & 50 & $0 \%$ & $5.5 \%$ \\
\hline & & & 60 & $20 \%$ & $27.5 \%$ \\
\hline
\end{tabular}

The students' distributions do not fit with the mixed NE distributions. The main difference concerns low bids different from 0 . The probabilities assigned to low bids ( 1 and 2 in Game 1, 10 and 20 in Game 2) by the NE are much higher than the frequencies with which the students play these bids: bids 1 and 2 in Game 1 are played with probability $34.9 \%$ in the NE, with probability $11.2 \%$ by the students; bids 10 and 20 in Game 2 are played with probability $34.54 \%$ in the NE, with probability $8.3 \%$ by the students. According to the 
comments added by the students, they fear that if they play low bids different from 0 , the opponent will bid more and make money whereas they lose money. In some way, bidding a low amount is seen as a way to encourage the opponent to bid more, even if there is no sequentiality in this game.

Another difference concerns the probability assigned to the value of the prize. Students bid this value much more often than in the NE $(20.7 \%$ versus $9.2 \%$ in the first game, $21.1 \%$ versus $11.1 \%$ in the second game).

We also observe that the students bid 0 more often than in the NE $(37.9 \%$ versus $28.3 \%$ in Game $1,33 \%$ versus $27.7 \%$ in Game 2), yet the difference in the probabilities in the second game is less significant.

The way students play bids higher than $\mathrm{V}$ is different in the two games. Whereas $30.2 \%$ of them play bids 4 and 5 almost with the same probabilities in Game 1 (in contrast to the NE that assigns probability 0 to bid 4 and $27.6 \%$ to bid 5), students, like the NE, assign a small probability to bids 40 and 50 in Game 2 (10.1\% for the students, $6.66 \%$ in the NE), and a large probability to bid 60 (27.5\% for the students, $20 \%$ in the NE).

It derives from these facts that the students' probabilities are different from the NE ones, and - this matters more - that the shapes of the students' distributions are quite different from the mixed NE one.

We claim that these differences simply highlight the fact that the philosophy of a mixed $\mathrm{NE}$ does not fit with the way of playing of real players. We justify this point of view by turning to best-reply matching.

\section{Philosophy of Mixed Nash Equilibria and Philosophy of Best-Reply Matching}

We first recall Kosfeld et al.'s [2002] Best-Reply Matching (BRM) equilibrium.

Definition 1. Kosfeld \& al. [2002]: Normal-form Best-Reply Matching equilibrium

Let $G=\left(\mathfrak{n}, S_{i},>_{i}, i \in \mathfrak{n}\right)$ be a game in normal form $\left(\mathfrak{n}\right.$ is the set of players, $N=$ Card,$S_{i}$ is player $i$ 's set of pure strategies, and $>_{i}$ is player $i$ 's preference relation on $\left.X_{i=1}^{N} S_{i}\right)$. A mixed strategy $p$ is a BRM equilibrium if for every player $i \in \mathcal{K}$ and for every pure strategy $s_{i} \in S_{i}:$

$$
p_{i}\left(s_{i}\right)=\sum_{s_{-i} \in B_{i}^{-1}\left(s_{i}\right)} \frac{1}{\operatorname{CardB}_{i}\left(s_{-i}\right)} p_{-i}\left(s_{-i}\right)
$$

where $B_{i}\left(s_{-i}\right)$ is the set of player i's best replies to the strategies $s_{-i}$ played by the other agents.

In a BRM equilibrium (BRME), the probability assigned to a pure strategy by player $\mathrm{i}$ is linked to the probability assigned to the opponents' strategies to which this pure strategy is a best reply: if player $\mathrm{i}^{\prime} \mathrm{s}$ opponents play $\mathrm{S}_{-\mathrm{i}}$ with probability $\mathrm{p}_{-\mathrm{i}}\left(\mathrm{s}_{-\mathrm{i}}\right)$, and if the set of 
player i's best responses to $\mathrm{s}_{-\mathrm{i}}$ is the subset of pure strategies $\mathrm{B}_{\mathrm{i}}\left(\mathrm{s}_{-\mathrm{i}}\right)$, then each strategy of this subset is played with probability $\mathrm{p}_{-\mathrm{i}}\left(\mathrm{s}_{-\mathrm{i}}\right)$ divided by the cardinal of $\mathrm{B}_{\mathrm{i}}\left(\mathrm{s}_{-\mathrm{i}}\right)$.

This criterion builds on the notion of rationalizability developed by Bernheim [1984] and Pearce [1984], a strategy $s_{i}$ being rationalizable if it is a best response to at least one profile S-i played by the other players. Kosfeld et al. [2002] simply observe that, if the opponents often play $\mathrm{S}_{-i}$, then $\mathrm{S}_{\mathrm{i}}$ often becomes the best response, so should often be played. In some way, they rationalize the probabilities of a player by the other players' probabilities.

We illustrate the concept on the normal-form game in matrix 3 .

\section{Player 2}

Player 1 $\quad \mathrm{A}_{1} \quad \mathrm{~A}_{2} \quad \mathrm{~B}_{2}$

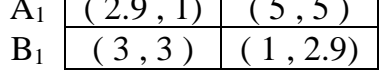

Matrix 3.

We call $p$ and $1-p$, respectively $q$ and $1-q$, the probabilities assigned to $A_{1}$ and $B_{1}$, respectively to $\mathrm{A}_{2}$ and $\mathrm{B}_{2}$. In the mixed $\mathrm{NE}$ we equalize player 1 's payoffs ${ }^{\mathrm{d}}$ obtained with $\mathrm{A}_{1}$ and $\mathrm{B}_{1}$, so we set $2.9 q+5(1-q)=3 q+1-q$, and we get $q=40 / 41$, i.e. a condition on player 2 's probabilities. We also equalize player 2's payoffs obtained with $\mathrm{A}_{2}$ and $\mathrm{B}_{2}, p+3(1-p)$ and $5 p+2.9(1-p)$, so we get $p=1 / 41$, a condition on player 1 's probabilities. This may seem quite strange from a behavioral point of view: a player's probabilities have no impact on his own payoff, they only ensure that the opponent is indifferent between his actions in the NE support. So, when player 1 plays $A_{1}$ with probability $1 / 41$ and $B_{1}$ with probability $40 / 41$, these probabilities mean nothing for herself. She could play $A_{1}$ and $B_{1}$ with any probabilities given that, due to player 2's probabilities, she is indifferent between $A_{1}$ and $\mathrm{B}_{1}$. She chooses probabilities $1 / 41$ and $40 / 41$ only to help player 2 to become indifferent between his two actions. This explains why she plays $\mathrm{B}_{1}$ with a probability close to 1 , even though $B_{1}$ is not interesting for her when considering the range of payoffs ( 2.9 and 5 for $A_{1}, 3$ and 1 for $B_{1}$ ). As a matter of fact, player 2's payoffs- when he plays $A_{2}$ and $B_{2-}$ are close when she plays $B_{1}$ (he gets 3 with $A_{2}$ and 2.9 with $B_{2}$ ) whereas they are quite different when she plays $A_{1}$ (he gets 1 with $A_{2}$ and 5 with $B_{2}$ ). And vice versa for player 2 .

We think that real players do not choose probabilities in this way. Especially if the support of the mixed NE is the whole set of pure strategies, have you ever seen a player who says: "let's try to put probabilities on my pure strategies so that the opponent gets the same payoff with all his pure strategies"? ${ }^{\mathrm{e}}$

\footnotetext{
d By 'payoff' we mean 'expected payoff', for all concepts of equilibria. For ease of notations, we will proceed so right through the paper.

e We do not say that this way of playing is always meaningless. If, by doing so, the payoff of the opponent is always low regardless of what he plays, and if the game is a zero-sum game (so a player is better off when his opponent is worse off) then behaving in such a way may be strategically meaningful. But, in a usual game like the one in matrix 3 , this behaviour is quite strange.
} 
In real life, behavior is less sophisticated (and less strange). When somebody plays A with probability $1 / 41$ and B with probability $40 / 41$, it is because he thinks that B is much more often his best response than A, 40 times more often, which justifies that he plays B with probability 40/41. In real life, probabilities (often) simply translate the frequency with which an action is supposed to be a best response, and, as a consequence, the frequency with which a player is ready to play it. And this is what is done in the BRME. Players simply try to be consistent with the way other players are playing, adapting their probability of playing an action to the probabilities with which the others play the actions to which this action is a best reply. This way of dealing with probabilities has no link with the mixed NE way of dealing with probabilities.

We define the BRME for the game in matrix 3:

$\mathrm{A}_{1}$ is player 1's best response to $\mathrm{B}_{2}$, so has to be played as often as $\mathrm{B}_{2}$, i.e. $p=1-q$.

$\mathrm{B}_{1}$ is player 1's best response to $\mathrm{A}_{2}$, so has to be played as often as $\mathrm{A}_{2}$, i.e. $1-p=q$.

$\mathrm{A}_{2}$ is player 2's best response to $\mathrm{B}_{1}$, so has to be played as often as $\mathrm{B}_{1}$, i.e. $q=1-p$.

$\mathrm{B}_{2}$ is player 2's best response to $\mathrm{A}_{1}$, so has to be played as often as $\mathrm{A}_{1}$, i.e. $1-q=p$.

And $0 \leq p \leq 1,0 \leq q \leq 1$. So, for the game studied, we get an infinite number of BRME, characterized by the fact that player 1 plays $A_{1}$ as often as player 2 plays $B_{2}$, and plays $B_{1}$ as often as player 2 plays $\mathrm{A}_{2}$ and vice versa.

Three remarks derive from these results:

- First, the BRM way of defining probabilities allows us to cope with the asymmetric pure strategy NE. Given that $A_{1}$ is the best response to $B_{2}$ and $B_{2}$ is the best response to $A_{1}$, the BRME allows player 1 to play $A_{1}$ with probability 1 and player 2 to play $B_{2}$ with probability 1 , given that $A_{1}$ is player 1 's best response as often as player 2 plays $\mathrm{B}_{2}$ and vice versa. A similar reasoning holds for the profile $\left(B_{1}, A_{2}\right)$. So, in this game, the pure strategy NE are also BRME.

- Second, the payoff in the mixed NE (here 121/41) may be higher or lower than the payoff in the BRME. In the game studied, as long as we choose $\mathrm{p}$ between 1/41 and 20/41, the players get more with the mixed NE than with the BRME, but for $\mathrm{p}$ lower than $1 / 41$ and $\mathrm{p}$ larger than 20/41, the players get more with the BRME.

- Third, in the game studied, the mixed NE is also a BRME (because $p=1-q=1 / 41$ ). Most often, mixed NE are not BRME. Observe that the justification of this special BRME is not the mixed NE one. In the BRME, player 1 plays $\mathrm{A}_{1}$ with probability $1 / 41$ because it is her best response to $B_{2}$ which is also played with probability $1 / 41$, and she plays $B_{1}$ with probability $40 / 41$ because it is her best response to $\mathrm{A}_{2}$, which is played with the same probability (and the symmetric explanation holds for player 2). So both actions are played because each is a best response, and not because they lead to the same payoff. In some degree, even when the BRME is a mixed equilibrium, players reason in a pure strategy way: in our example the aim is to play A when the other plays B, and to play B when he plays A. This is not the case in a mixed NE, where each player best reacts to the mixed strategies of the others. 
In the paper we mainly focus on a generalization of the BRM concept. As a matter of fact, when there are several best replies to a profile $s_{-i}$, there is no reason to demand that each best reply be played with the same probability, so it is reasonable to generalize Kosfeld et al.'s criterion by allowing players to play the different best replies with different probabilities.

Definition 2. Umbhauer [2016]: Normal-form Generalized Best-reply Matching equilibrium. Let $G=\left(\mathfrak{n}, S_{i},>_{i}, i \in \mathfrak{N}\right)$ be a game in normal form. A mixed strategy $p$ is a Generalized BRM (GBRM) equilibrium iffor every player $i \in \mathbb{N}$ and for every pure strategy $s_{i} \in S_{i}$ :

$$
p_{i}\left(s_{i}\right)=\sum_{s_{-i} \in B_{i}^{-1}\left(s_{i}\right)} \delta_{s_{i} s_{-i}} p_{-i}\left(s_{-i}\right)
$$

with $\delta_{s_{i} s_{-i}} \in[0,1]$ for any $s_{i}$ belonging to $B_{i}\left(s_{-i}\right)$ and $\sum_{s_{i} \in B_{i}\left(s_{-i}\right)} \delta_{s_{i} s_{-i}}=1$.

Pure NE, in contrast to mixed ones, are automatically GBRM equilibria (GBRME): if player 1 plays A and player 2 plays B in a pure strategy NE -so they play the actions with probability 1-, player 1 plays A as often as the opponent plays the action B to which A is a -perhaps among several- best reply, and player 2 plays B as often as player 1 plays the action A to which B is a -perhaps among several- best reply (Umbhauer [2016]).

We make two additional remarks. Firstly, BRME and GBRME are ordinal concepts. This may prevent a good strategy, for example a cautious strategy that leads to high payoffs but is never a best response to the pure strategy profiles of the opponents, from being played in a BRME or in a GBRME. This is not intuitive and allows us to conjecture that BRM behavior will not always be observed in real life (in real life, players like playing cautious strategies that lead to high payoffs even when these strategies are neither best responses to the pure strategy profiles of the opponents, nor belong to a NE). Yet in the second-price all-pay auction, there do not exist strategies that lead to high payoffs without being a best response. Bidding 0 is a cautious way of playing but it leads to a positive net payoff only if the other player bids 0 too. Secondly, the second-price all-pay auction has a special structure as regards best responses : there are many best responses to a given bid (when it is not higher than V), so that GBRM becomes very useful and powerful; this will be developed in the following sections.

\section{Best-Reply Matching and Students' Way of Playing}

We establish the BRME in the second-price all-pay auctions.

We start with the games played by the students. To do so, we write the best-reply matrices 4a (Game 1) and 4b (Game 2), where $b_{i}$ means that player i's action is a best reply to the 
opponent's action, $i=1,2$. For example, the bold $\mathrm{b}_{1}$ in italics in matrix $4 \mathrm{a}$ means that bid 4 is one of player 1's best replies to player 2's bid 1 .

\section{Player 2}

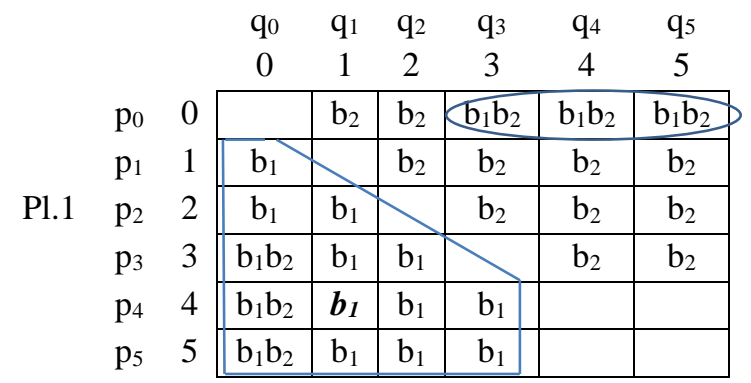

Matrix4a. Best-reply Matrix Game 1.

\section{Player 2}

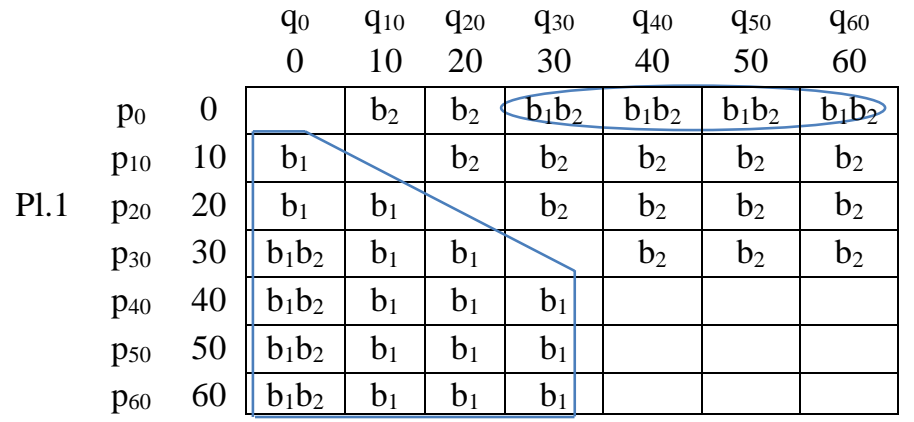

Matrix 4b. Best-reply Matrix Game 2.

It derives from the matrices $4 \mathrm{a}$ and $4 \mathrm{~b}$ that the best responses in the second-price all-pay auction match a special structure: each bid $\mathrm{x}$ higher than 0 and up to $\mathrm{V}$ is a best response to each bid lower than $\mathrm{x}$, each bid higher than $\mathrm{V}$ is a best response to all bids from 0 to $\mathrm{V}$, and 0 is a best response to each bid from $\mathrm{V}$ to $\mathrm{M}$. This fact is illustrated for player 1 in both matrices: the diagrams contain player 1's best responses.

In all the games studied, we write $\mathrm{p}_{\mathrm{i}}$, respectively $\mathrm{q}_{\mathrm{i}}$, the probability assigned to bid $\mathrm{i}$ by player 1 , respectively by player 2 .

In Game $1^{\mathrm{f}}$, i goes from 0 to 5 . The symmetry of the game leads to the symmetric set of equations:

$$
\begin{array}{ll}
p_{0}=q_{3} / 3+q_{4}+q_{5} & q_{0}=p_{3} / 3+p_{4}+p_{5} \\
p_{1}=q_{0} / 5 & q_{1}=p_{0} / 5 \\
p_{2}=q_{0} / 5+q_{1} / 4 & q_{2}=p_{0} / 5+p_{1} / 4 \\
p_{3}=q_{0} / 5+q_{1} / 4+q_{2} / 3 & q_{3}=p_{0} / 5+p_{1} / 4+p_{2} / 3 \\
p_{4}=q_{0} / 5+q_{1} / 4+q_{2} / 3+q_{3} / 3 & q_{4}=p_{0} / 5+p_{1} / 4+p_{2} / 3+p_{3} / 3
\end{array}
$$

\footnotetext{
${ }^{\mathrm{f}}$ For some results of Game 1, see also Umbhauer [2016].
} 
$\begin{array}{ll}p_{5}=q_{0} / 5+q_{1} / 4+q_{2} / 3+q_{3} / 3=p_{4} & q_{5}=p_{0} / 5+p_{1} / 4+p_{2} / 3+p_{3} / 3=q_{4} \\ p_{0}+p_{1}+p_{2}+p_{3}+p_{4}+p_{5}=1 & q_{0}+q_{1}+q_{2}+q_{3}+q_{4}+q_{5}=1 .\end{array}$

This system of equations has a unique solution: $p_{0}=q_{0}=180 / 481=37.4 \%$, $p_{1}=q_{1}=p_{0} / 5=7.5 \%, p_{2}=q_{2}=p_{0} / 4=9.4 \%, p_{3}=q_{3}=p_{0} / 3=12.5 \%, p_{4}=p_{5}=q_{4}=q_{5}=4 p_{0} / 9=16.6 \%$.

These results are reproduced in Table 3 .

Table 3. BRME for Game 1, Game 2 and $(\mathrm{V}=9, \mathrm{M}=12)^{\mathrm{a}, \mathrm{b}, \mathrm{c}}$.

\begin{tabular}{|l|l|l|l|l|l|l|l|l|l|l|l|}
\hline & $\mathrm{q}_{0}$ & $\mathrm{q}_{1}$ & $\mathrm{q}_{2}$ & $\mathrm{q}_{3}$ & $\mathrm{q}_{4}$ & $\mathrm{q}_{5}$ & $\mathrm{q}_{6}$ & $\mathrm{q}_{7}$ & $\mathrm{q}_{8}$ & $\mathrm{q}_{9}$ & $\begin{array}{l}\mathrm{q}_{10}, \mathrm{q}_{11}, \\
\mathrm{q}_{12}\end{array}$ \\
\hline $\mathrm{V}=3$ & $180 /$ & $36 /$ & $45 /$ & $60 /$ & $80 /$ & $80 /$ & & & & & \\
$\mathrm{M}=$ & $481=$ & $481=$ & $481=$ & $481=$ & $481=$ & $481=$ & & & & & \\
5 & 0.374 & 0.075 & 0.094 & 0.125 & 0.166 & 0.166 & & & & & \\
$*$ & & & & & & & & & & & \\
$\mathrm{~V}=$ & $240 /$ & $40 /$ & $48 /$ & $60 /$ & $75 /$ & $75 /$ & $75 /$ & & & & \\
30 & $613=$ & $613=$ & $613=$ & $613=$ & $613=$ & 613 & 613 & & & & \\
$\mathrm{M}=$ & 0.3915 & 0.065 & 0.078 & 0.098 & 0.1225 & & & & & & \\
60 & & & & & & & & & & & \\
$*$ & & & & & & & & & & \\
\hline $\mathrm{V}=9$ & 55440 & 4620 & 5040 & 5544 & 6160 & 6930 & 7920 & 9240 & 11088 & 13860 & 17325 \\
$\mathrm{M}=$ & $/ \mathrm{D}=$ & $/ \mathrm{D}=$ & $/ \mathrm{D}=$ & $/ \mathrm{D}=$ & $/ \mathrm{D}=$ & $/ \mathrm{D}=$ & $/ \mathrm{D}=$ & $/ \mathrm{D}=$ & $/ \mathrm{D}=$ & $/ \mathrm{D}=$ & $/ \mathrm{D}=$ \\
12 & 0.312 & 0.026 & 0.0285 & 0.031 & 0.035 & 0.039 & 0.045 & 0.052 & 0.0625 & 0.078 & 0.097 \\
$*$ & & & & & & & & & & & \\
\hline
\end{tabular}

a The notations q0, q1, q2, q3, q4, q5, q6 should be read q0, q10, q20, q30, q40, q50, q60 for V=30 and M=60. $\mathrm{b} *=$ unique BRME

${ }^{\mathrm{c}} \mathrm{D}=177817$

Game 2 leads to the equations:

$$
\begin{array}{ll}
p_{0}=q_{30} / 4+q_{40}+q_{50}+q_{60} & q_{0}=p_{30} / 4+p_{40}+p_{50}+p_{60} \\
p_{10}=q_{0} / 6 & q_{10}=p_{0} / 6 \\
p_{20}=q_{0} / 6+q_{10} / 5 & q_{20}=p_{0} / 6+p_{10} / 5 \\
p_{30}=q_{0} / 6+q_{10} / 5+q_{20} / 4 & q_{30}=p_{0} / 6+p_{10} / 5+p_{20} / 4 \\
p_{40}=q_{0} / 6+q_{10} / 5+q_{20} / 4+q_{30} / 4 & q_{40}=p_{0} / 6+p_{10} / 5+p_{20} / 4+p_{30} / 4 \\
p_{50}=q_{0} / 6+q_{10} / 5+q_{20} / 4+q_{30} / 4=p_{40} & q_{50}=p_{0} / 6+p_{10} / 5+p_{20} / 4+p_{30} / 4=q_{40} \\
p_{60}=q_{0} / 6+q_{10} / 5+q_{20} / 4+q_{30} / 4=p_{40} & q_{60}=p_{0} / 6+p_{10} / 5+p_{20} / 4+p_{30} / 4=q_{40} \\
p_{0}+p_{10}+p_{20}+p_{30}+p_{40}+p_{50}+p_{60}=1 & q_{0}+q_{10}+q_{20}+q_{30}+q_{40}+q_{50}+q_{60}=1 .
\end{array}
$$

The unique solution, reproduced in Table 3 , is $p_{0}=q_{0}=240 / 613=39.15 \%$, $p_{10}=q_{10}=p_{0} / 6=40 / 613=6.5 \%, p_{20}=q_{20}=p_{0} / 5=48 / 613=7.8 \%, p_{30}=q_{30}=p_{0} / 4=60 / 613=9.8 \%$, $p_{40}=p_{50}=p_{60}=q_{40}=q_{50}=q_{60}=5 p_{0} / 16=75 / 613=12.25 \%$.

More generally, due to the structure of the second-price all-pay auction, there is a unique BRME, given in proposition 1.

Proposition 1. For $M \geq V$ and $M>1$ the unique BRME is symmetric and given by:

$$
\begin{array}{lll}
q_{0}=\frac{1}{2+\sum_{i=0}^{V-1} \frac{1}{M-i}-\frac{1}{(M-V+1)^{2}}} & & \\
q_{i}=q_{0} /(M-i+1) & & \text { ifrom } 1 \text { to } V, \\
q_{i}=q_{0}(M-V+2) /(M-V+1)^{2} & & \text { ifrom } V+1 \text { to } M(\text { when } M>V),
\end{array}
$$


where $q_{i}$ is the probability of playing bid $i$, i from 0 to $M$.

We get: $\sum_{i=V+1}^{M} q_{i}<q_{0}<\sum_{i=V}^{M} q_{i}$ if $M>V$ and $q_{0}=q_{V}$ if $M=V$.

For $M=V=1$ there exist an infinite number of $B R M E$ defined by: $q_{1}=p_{0}, p_{0}+p_{1}=1, q_{0}+q_{1}=1$.

Proof see Appendix C.

Proposition 1 allows us to establish the BRME for $\mathrm{V}=9$ and $\mathrm{M}=12$. The probabilities are given in Table 3. The BRME are given in Fig. 2a, 2b and 2c.

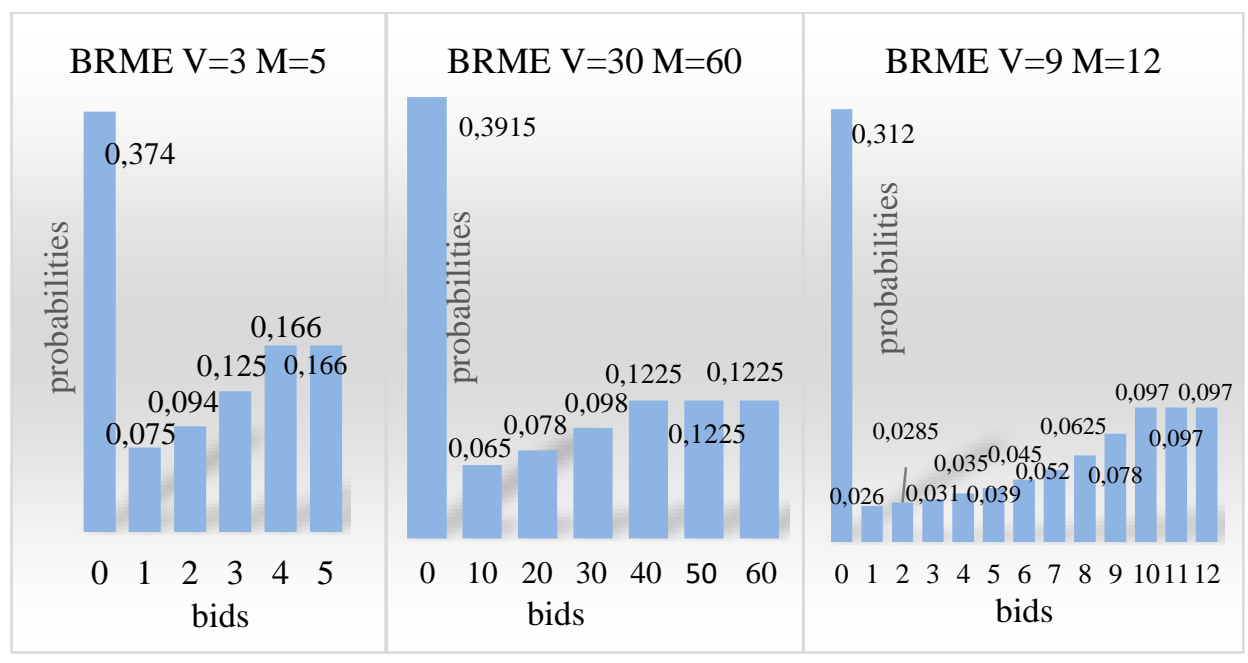

Fig. 2a. $B R M E(V=3, M=5)$.

Fig. 2b. $\operatorname{BRME}(\mathrm{V}=30, \mathrm{M}=60)$.

Fig. 2c. $\mathrm{BRME}(\mathrm{V}=9, \mathrm{M}=12)$.

We first compare the shape of the BRME distribution and the shape of the mixed NE distribution.

In the BRME, $\mathrm{q}_{\mathrm{i}}$ is increasing in $\mathrm{i}$ for $\mathrm{i}$ from 1 to $\mathrm{V}+1$, and is constant from $\mathrm{V}+1$ to $\mathrm{M}$, a result in sharp contrast with the mixed NE probabilities that are decreasing from 1 to $\mathrm{M}$ $\mathrm{V} / 2-1 / 2$ and null from $\mathrm{M}-\mathrm{V} / 2+1 / 2$ to $\mathrm{M}-1$. BRM clearly takes into account that a higher bid is more often a best reply than a lower one (different from 0 ), in that each bid $x$ (different from 0 ) is a best reply to all the bids lower than $\mathrm{x}$, if $x \leq V$, and a best-reply to all bids from 0 to $\mathrm{V}$ if $x>V$. And bid 0 , in contrast to the other low bids, has a special status in that it is a best reply to all bids from $\mathrm{V}$ to $\mathrm{M}$.

Clearly, the Nash and BRM distributions have no common points, except the fact that the probability on bid 0 is higher than the probabilities assigned to the bids higher than 0 and lower than or equal to $\mathrm{V}$, both in the BRME and in the NE (when $M>V$ ). The strong differences and the few similarities are highlighted by comparing two by two Fig. 1a and $2 \mathrm{a}, 1 \mathrm{~b}$ and $2 \mathrm{~b}, 1 \mathrm{c}$ and $2 \mathrm{c}$.

But what about BRM and the student's behavior?

Fig. $3 \mathrm{a}, 3 \mathrm{~b}$ and $3 \mathrm{c}$ give the students' behavior, the NE and the BRME in Game 1. 


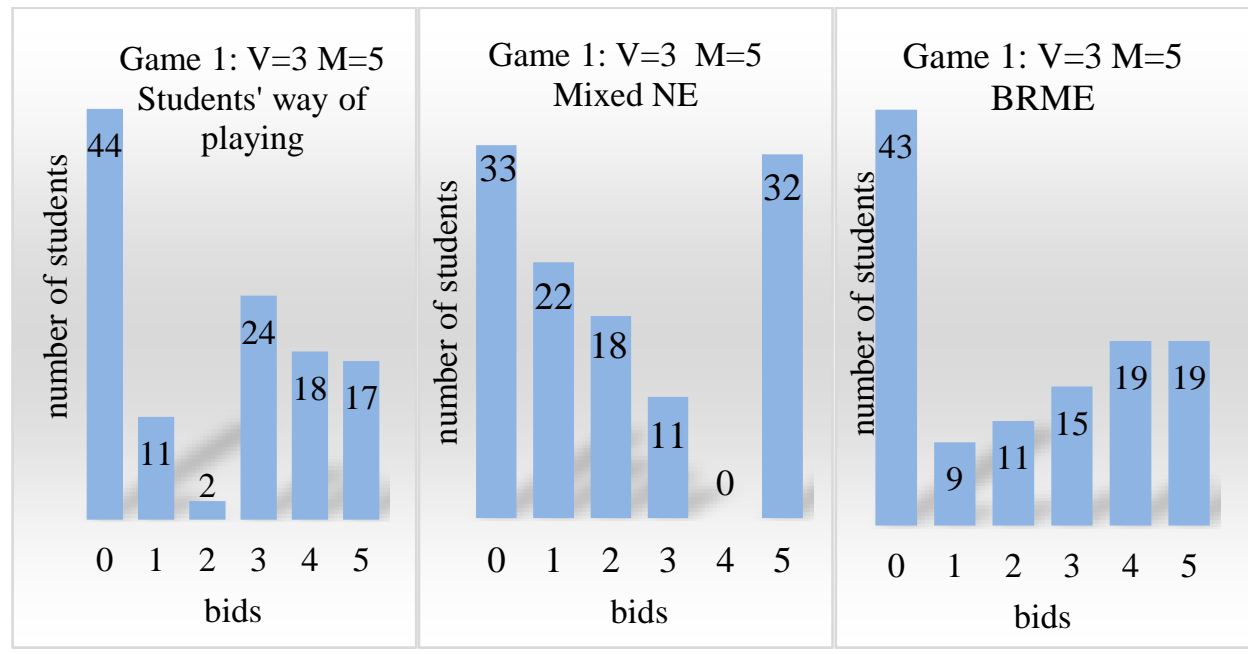

Fig. 3a. Game1, Students' way of playing. Fig. 3b. NE Game 1.

Fig. 3c. BRME Game 1.

In Game 1, the BRME probabilities fit much better with the students' probabilities, except $\mathrm{p}_{2}$ (higher) and $\mathrm{p}_{3}$ (lower). This proximity is due to the fact that BRM exploits some observations made by the students in their comments, namely that bids 1 and 2 are seldom best responses. In fact, bid 1 is a best response only if the opponent bids 0 (and in this case, bids 2, 3, 4, 5 are also best responses), bid 2 is a best response only if the opponent bids 0 or 1 and in these two cases, bids 3, 4 and 5 are also best responses. By contrast, 0, 3, 4 and 5 are often best responses (bid 0 is the unique best response to bids 4 and 5 and one best response to bid 3, bid 3 is a best response to bids 0,1 and 2, bids 4 and 5 are best responses to bids $0,1,2$ and 3 ).

We turn to Game 2: Fig. 4a, 4b and 4c give the students' behavior, the NE and the BRME. This time, both the BRME and the NE are strongly different from the students' behavior. This can be explained by the fact that the students, especially when the number of bids grows and when there are several best replies, do not play all best replies with the same probability, and may even choose to play only some of them, as allowed by GBRM. 


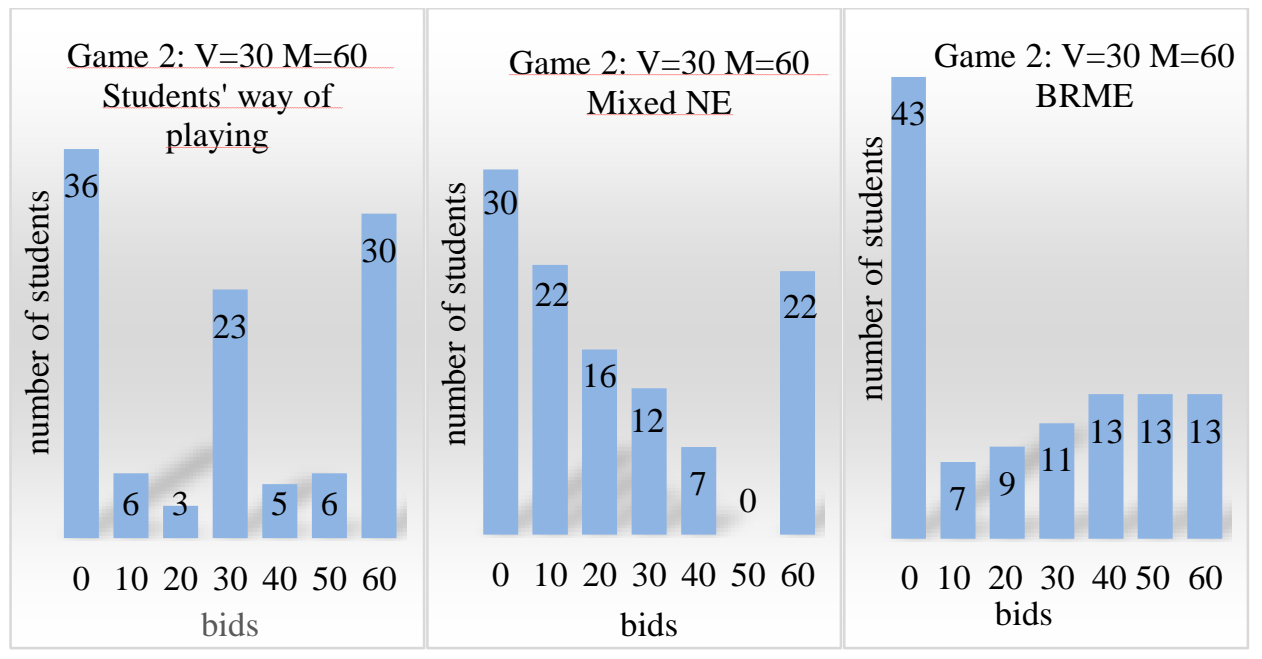

Fig. 4a. Game 2, Students' way of playing. Fig. 4b. NE Game $2 . \quad$ Fig. 4c. BRME Game 2.

\section{Generalized Best-Reply Matching, Focal Values and Focal Behavior, a Way to bring closer First-Price and Second-Price All-Pay Auctions}

In a second-price all-pay auction, all the bids higher than $\mathrm{x}$ and up to $\mathrm{M}$ are best responses to $\mathrm{x}$, when $\mathrm{x}$ is not exceeding $\mathrm{V}$. It is very reasonable to expect that players will not play each best response with the same probability but may prefer playing best responses that are focal, that is to say that have something special that make them prominent. That is why GBRM fits better with real behavior. So, for example, in Game 2, bids 40, 50 and 60 are best replies to bids $0,10,20$ and 30, which explains that they are each played with the same probability 0.1225 in the BRME. Yet 40 and 50 are much less focal than 60 , given that 60 , besides being a best response, has focal properties not shared by 40 and 50: 60 is the largest possible bid, so a player is sure to get the prize at least with probability $1 / 2$ by bidding 60 . This may lead a player to only (mostly) playing 60, instead of playing 40, 50 and 60 with the same probability 0.1225 (this does not change the other probabilities in the system of equations). So, according to GBRM, the player can play 40 and 50 with a small probability, and 60 with the probability complementary to $3 \mathrm{X} 0.1225=0.3675$. And this is exactly what is done by the students; they play 40 with probability $0.046,50$ with probability 0.055 and 60 with probability 0.275 , which leads to the total amount 0.376 , a probability which is close to 0.3675 .

In Game 2, 3 bids are more focal than others: 0 , because it is the cautious bid ${ }^{\mathrm{g}}, \mathrm{V}$, because it is the value of the prize, hence the fair price to pay, and $\mathrm{M}$, which is the highest possible bid and which leads to getting the prize at least with probability $1 / 2$.

${ }^{\mathrm{g}} 0$ is sometimes also viewed as a cooperative bid, because bidding 0 allows a player to get the prize half of the time without paying anything, when both players bid 0 . 
So suppose that the students prefer playing focal values each time they belong to the best responses. For example, when player 1 bids 10, player 2 only best replies with bid 30 and bid 60 , even though bids 20, 40 and 50 are also best responses. This leads to the GBRM matrix 5 (consider only the $b_{1}$ and $b_{2}$, the $B_{1}$ and $B_{2}$ are used in a further study).

Player 2

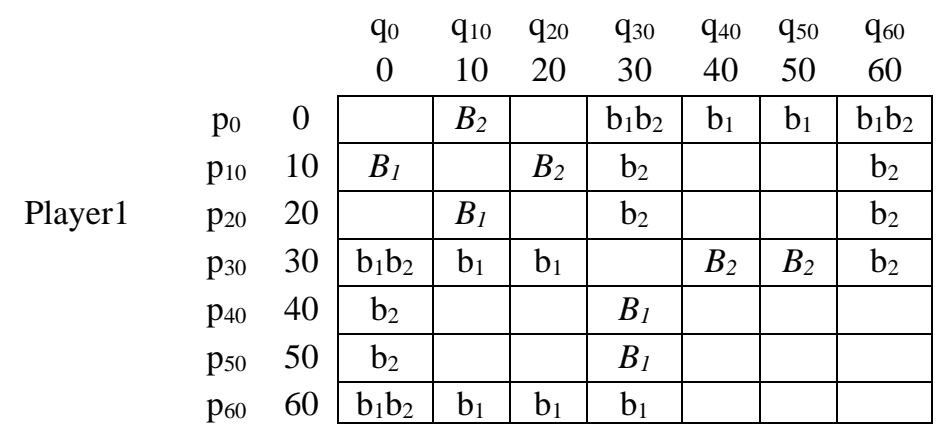

Matrix 5. Selected best responses in Game 2.

The system of equations becomes:

$$
\begin{array}{ll}
p_{0}=q_{30} / 2+q_{40}+q_{50}+q_{60} & q_{0}=p_{30} / 2+p_{40}+p_{50}+p_{60} \\
p_{10}=0 & q_{10}=0 \\
p_{20}=0 & q_{20}=0 \\
p_{30}=q_{0} / 2+q_{10} / 2+q_{20} / 2 & q_{30}=p_{0} / 2+p_{10} / 2+p_{20} / 2 \\
p_{40}=0 & q_{40}=0 \\
p_{50}=0 & q_{50}=0 \\
p_{60}=q_{0} / 2+q_{10} / 2+q_{20} / 2+q_{30} / 2 & q_{60}=p_{0} / 2+p_{10} / 2+p_{20} / 2+p_{30} / 2 \\
p_{0}+p_{10}+p_{20}+p_{30}+p_{40}+p_{50}+p_{60}=1 & q_{0}+q_{10}+q_{20}+q_{30}+q_{40}+q_{50}+q_{60}=1 .
\end{array}
$$

The unique GBRME, solution of this system of equations, is: $p_{0}=q_{0}=4 / 9=44.4 \%$ $p_{30}=q_{30}=p_{0} / 2=22.2 \%, p_{60}=q_{60}=3 p_{0} / 4=33.3 \% p_{10}=q_{10}=p_{20}=q_{20}=p_{40}=q_{40}=p_{50}=q_{50}=0$.

We get a 3 peak distribution which is similar to that of the students as regards the shape (highest peak on 0 , second highest peak on 60 and lowest peak on 30) (see Table 4 and Fig. 5a).

To focal bids we can add focal behavior. We suggest that the players, among the multiple best responses, may prefer choosing responses that fit with a type of behavior. For example, they may add cautiousness to best replying. So, when the opponent bids 0 , the bids 10,20 , $30,40,50$ and 60 are best responses, but they choose 10 because 10 is the most cautious bid in the set of best responses (in that it maximizes the minimum payoff in this set, the minimum payoffs being respectively 50, 40, 30, 20, 10 and 15 for bids 10, 20, 30, 40, 50 and 60). In the same way, 20 is the most cautious best reply to 10 and 30 is the most cautious best response to 20. So cautiousness in some groups of players (and other types 
of behavior in other groups of players) may help them to select actions in the set of best responses.

\begin{tabular}{ccccc}
\hline $\begin{array}{c}\text { V=30, } \\
\begin{array}{c}\text { M=60 } \\
\text { bids }\end{array}\end{array}$ & $\begin{array}{c}\text { Tash } \\
\text { equilibrium }\end{array}$ & $\begin{array}{c}\text { Students' } \\
\text { behavior }\end{array}$ & $\begin{array}{c}\text { GBRME } \\
\text { with bids 0, 30 } \\
\text { and 60 }\end{array}$ & $\begin{array}{c}\text { GBRME with } \\
\text { weighted focal } \\
\text { values }\end{array}$ \\
\hline $\mathbf{0}$ & $27.7 \%$ & $33 \%$ & $44.5 \%$ & $35.5 \%$ \\
\hline $\mathbf{1 0}$ & $20.48 \%$ & $5.5 \%$ & 0 & $11.8 \%$ \\
\hline $\mathbf{2 0}$ & $14.06 \%$ & $2.8 \%$ & 0 & $3.9 \%$ \\
\hline $\mathbf{3 0}$ & $11.1 \%$ & $21.1 \%$ & $22.2 \%$ & $17.7 \%$ \\
\hline $\mathbf{4 0}$ & $6.66 \%$ & $4.6 \%$ & 0 & $4.45 \%$ \\
\hline $\mathbf{5 0}$ & $0 \%$ & $5.5 \%$ & 0 & $4.45 \%$ \\
\hline $\mathbf{6 0}$ & $20 \%$ & $27.5 \%$ & $33.3 \%$ & $22.2 \%$ \\
\hline
\end{tabular}

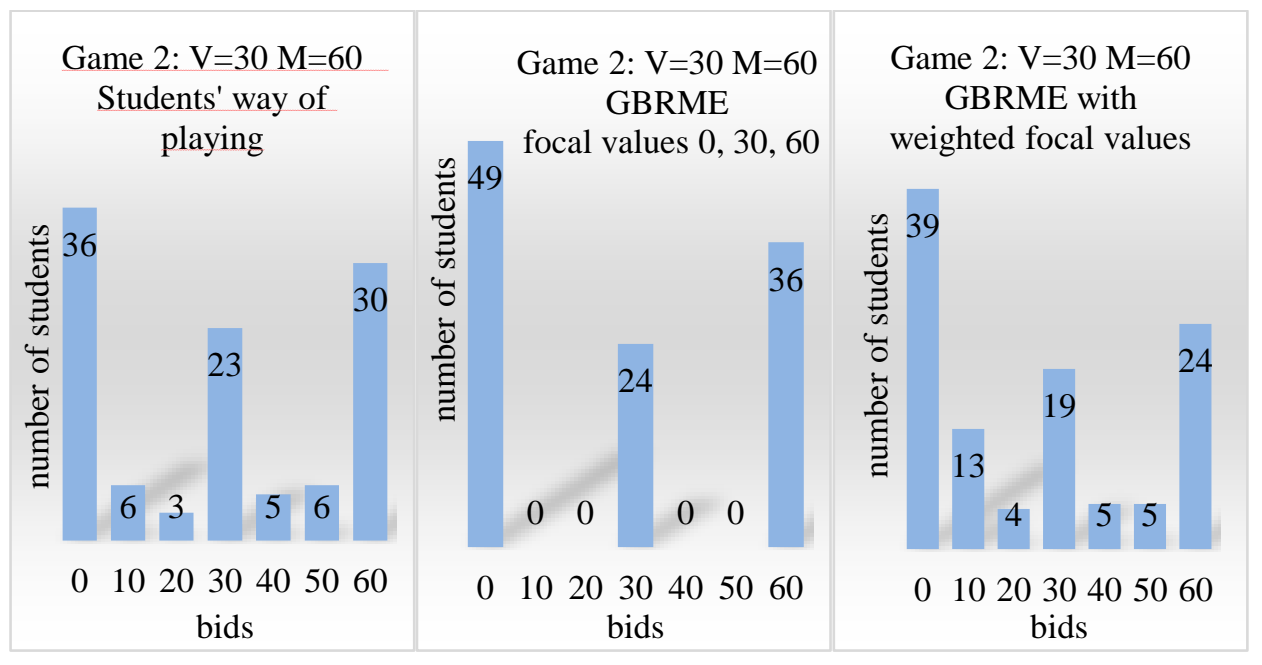

Fig. 4a. Game 2 Students' way

Fig. 5a. GBRME Game 2 of playing.

Fig. 5b. GBRME Game 2 weighted focal values.

To come closer to the students' distribution, we add some cautious behavior (in addition to the play of the three focal values $0, \mathrm{~V}$ and $\mathrm{M}$ ), by assuming that players also focus on 10 as the cautious best response to 0 , and on 20 as the cautious best response to 10 . And we also add 40 and 50 as best responses to 30 (perhaps because some players like outbidding the fair price bidders, without necessarily focusing exclusively on 60). So we add the $\mathrm{B}_{1}$ and $\mathrm{B}_{2}$ in matrix 5 and we get the equations:
$p_{0}=q_{30} / 4+q_{40}+q_{50}+q_{60}$
$q_{0}=p_{30} / 4+p_{40}+p_{50}+p_{60}$
$p_{10}=q_{0} / 3$
$q_{10}=p_{0} / 3$
$p_{20}=q_{10} / 3$
$q_{20}=p_{10} / 3$ 


$$
\begin{array}{ll}
p_{30}=q_{0} / 3+q_{10} / 3+q_{20} / 2 & q_{30}=p_{0} / 3+p_{10} / 3+p_{20} / 2 \\
p_{40}=q_{30} / 4=p_{50} & q_{40}=p_{30} / 4=q_{50} \\
p_{60}=q_{0} / 3+q_{10} / 3+q_{20} / 2+q_{30} / 4 & q_{60}=p_{0} / 3+p_{10} / 3+p_{20} / 2+p_{30} / 4 \\
p_{0}+p_{10}+p_{20}+p_{30}+p_{40}+p_{50}+p_{60}=1 & q_{0}+q_{10}+q_{20}+q_{30}+q_{40}+q_{50}+q_{60}=1 .
\end{array}
$$

The unique solution of this system of equations is : $p_{0}=q_{0}=72 / 203=35.5 \%$, $p_{10}=q_{10}=24 / 203=11.8 \%, p_{20}=q_{20}=8 / 203=3.9 \%, p_{30}=q_{30}=36 / 203=17.7 \%, p_{40}=p_{50}=q_{40}=$ $q_{50}=9 / 203=4.45 \%$ and $p_{60}=q_{60}=45 / 203=22.2 \%$.

These values are illustrated in Fig. $5 \mathrm{~b}$ and in Table 4 . It derives from this that we can come close to the students' distribution both in probabilities (see Table 4) and in shape (see Fig. $5 \mathrm{~b}$ and Fig. 4a).

We now turn to the general discrete second-price all-pay auction $(\mathrm{V}, \mathrm{M}$ are integers and the bids are the integers from 0 to $M$ ) and show that we get a GBRME of special interest, in that it is also a GBRME of the first-price all-pay auction.

We recall that the only - but crucial - difference between a first-price all-pay auction and a second-price all-pay auction is that in the first-price all-pay auction each player pays his bid. So the structure of the best-reply matrix is different.

We show the first-price all-pay auction for $V=3$ and $M=6$ in matrix $6 a$.

\section{Player 2}

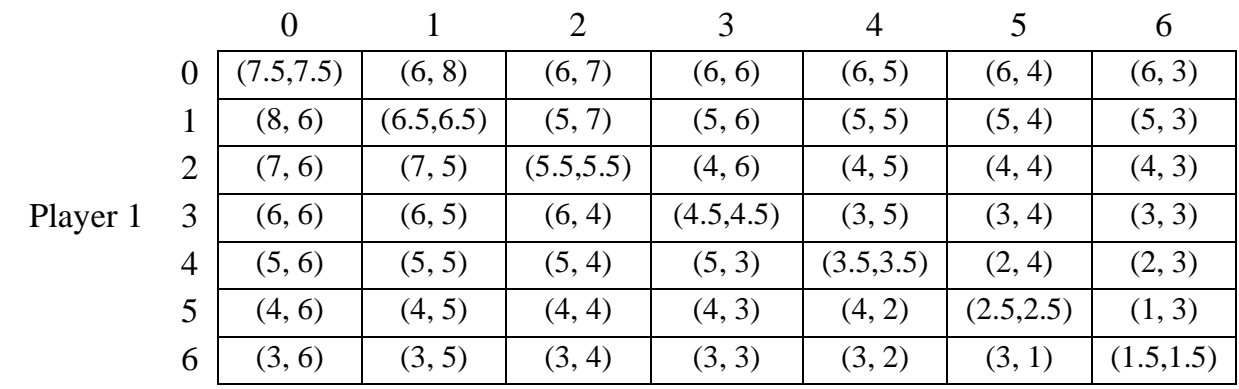

Matrix 6a. First -price all-pay auction $(\mathrm{V}=3, \mathrm{M}=6)$.

We recall in matrix $6 \mathrm{~b}$ the best-reply matrix for the second-price all-pay auction with $\mathrm{V}=3$ and $M=6$; matrix $6 \mathrm{c}$ is the best-reply matrix for the first-price all-pay auction with $\mathrm{V}=3$ and $\mathrm{M}=6$.

It derives from matrices $6 b$ and $6 c$ that, for any bid, the intersection of the sets of best responses to this bid, in the second-price all-pay auction, and in the first-price all-pay auction, is never empty. It follows from this that, by selecting the same best responses in the intersection, we necessarily get the same GBRME for both games.

So suppose that the players, in the second-price all-pay auction, among the best responses, only choose the most cautious one. It follows that each player best replies to the opponent's bid $x$ by playing the lowest possible best reply (because it is the bid that yields the maxmin payoff in the set of best responses). So bid $x+1$ is chosen as the best response to bid $x$, 
for $\mathrm{x}$ from 0 to $\mathrm{V}-1$, and bid 0 is chosen as the best response to all bids higher than or equal to $\mathrm{V}$.

Player 2

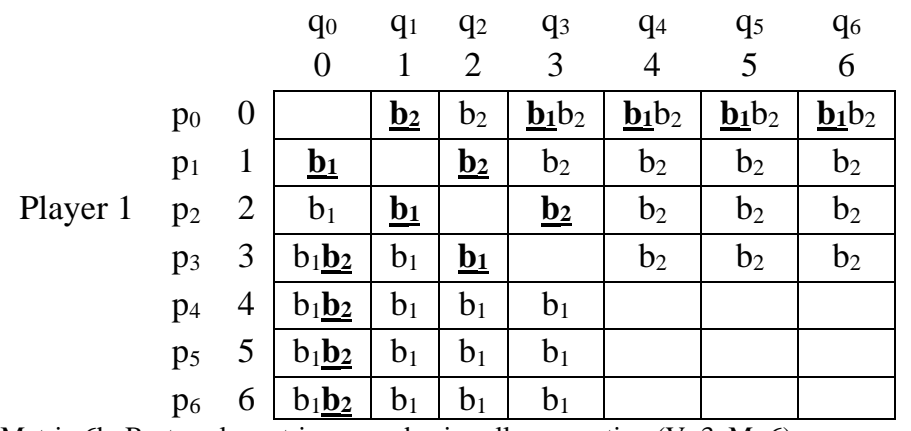

Matrix 6b. Best-reply matrix, second-price all-pay auction ( $\mathrm{V}=3, \mathrm{M}=6)$.

\section{Player 2}

\begin{tabular}{|c|c|c|c|c|c|c|c|c|c|}
\hline & & & $\begin{array}{c}\mathrm{q}_{0} \\
0\end{array}$ & $\begin{array}{c}\mathrm{q}_{1} \\
1\end{array}$ & $\begin{array}{l}\mathrm{q}_{2} \\
2\end{array}$ & $\begin{array}{l}\mathrm{q}_{3} \\
3\end{array}$ & $\begin{array}{l}\mathrm{q}_{4} \\
4\end{array}$ & $\begin{array}{c}\mathrm{q}_{5} \\
5\end{array}$ & $\begin{array}{c}\mathrm{q}_{6} \\
6\end{array}$ \\
\hline \multirow{7}{*}{ Player 1} & & 0 & & $\underline{\mathbf{b}_{2}}$ & $\mathrm{~b}_{1}$ & $\underline{\mathbf{b}}_{1}$ & $\underline{\mathbf{b}}_{1}$ & $\underline{\mathbf{b}}_{\mathbf{1}}$ & $\underline{\mathbf{b}_{1}}$ \\
\hline & & 1 & b1 & & $\underline{\mathbf{b}_{2}}$ & & & & \\
\hline & & 2 & $\mathrm{~b}_{2}$ & $\underline{\mathbf{b}_{1}}$ & & $\underline{\mathbf{b}_{2}}$ & & & \\
\hline & & 3 & $\underline{\mathbf{b}_{2}}$ & & $\underline{b_{1}}$ & & & & \\
\hline & & 4 & $\underline{\mathbf{b}_{2}}$ & & & & & & \\
\hline & & 5 & $\underline{\mathbf{b}_{2}}$ & & & & & & \\
\hline & $\mathrm{p}_{6}$ & 6 & $\underline{\mathbf{b}_{2}}$ & & & & & & \\
\hline
\end{tabular}

In the first-price all-pay auction, bid $\mathrm{x}+1$ is the only best response to bid $\mathrm{x}, \mathrm{x}$ from 0 to $\mathrm{V}$ 2 , bid 0 is the only best reply to all bids higher than or equal to $\mathrm{V}$, and bid 0 and bid $\mathrm{V}$ are the only best responses to bid V-1. Suppose that the players choose V as the best response to $\mathrm{V}-1$, - i.e. that they select the less cautious best response.

By so doing, in both games, the players choose for any bid the same best response (the bold and underlined best responses in matrices $6 b$ and $6 c$ ). So they play the same GBRME.

Given that $p_{i}=q_{i}=0$, for $\mathrm{i}$ from $\mathrm{V}+1$ to $\mathrm{M}$, the GBRM equations become:

$p_{0}=q_{V} \quad q_{0}=p_{V}$

$p_{i}=q_{i-1} \quad q_{i}=p_{i-1} \quad i$ from 1 to $\mathrm{V} \quad \sum_{i=0}^{V} p_{i}=\sum_{i=0}^{V} q_{i}=1$.

It follows that the unique symmetric GBRME assigns the same probability $1 /(\mathrm{V}+1)$ to each bid from 0 to $\mathrm{V}$ and a null probability to the higher bids.

So playing in a cautious (best-replying) way in the second-price all-pay auction leads to the same strategy profile as playing in a non-cautious (best-replying) way in the first-price all-pay auction. 
This result is of interest, both from a game theory point of view and from an economic point of view.

Firstly, in game theory, getting a same equilibrium behavior for two different games means that the equilibrium is robust to important changes in the game. And this is interesting given that players do often not perfectly understand the rules of a game. Especially, as regards all-pay auctions, which are special non obvious auctions, some players may be unsure about what they will have to pay: even if one takes time explaining the rules of a second-price all-pay auction, one can reasonably anticipate that some players keep on thinking that they pay what they bid. So some players play without knowing what they have to pay, i.e. they play a game with incomplete information on the payoffs. Hence it is fine to get an equilibrium that is robust to different payoff structures. In our approach, getting the same equilibrium in two different games is possible when the intersection of the sets of best responses to each action in both games is not empty, which restricts the ways in which the payoff structures are allowed to differ. This observation contributes to the literature on the robustness of equilibrium behavior in games with incomplete information (see for example Bergemann and Morris [2013]).

Secondly, it is sometimes difficult to distinguish a first-price all-pay auction from a secondprice all-pay auction. Consider for example Shubik's ${ }^{\text {h }}$ [1971] dollar auction game, where two bidders make bids and can outbid each other (in multiple of five cents) in order to get a dollar. The game stops when one bidder drops out; the dollar goes to the highest bidder, and both bidders pay the largest bid they made. This game is close to a first-price all-pay auction, in that each bidder pays his largest bid. But, because the game is sequential, one may observe a sequential overbidding process - the escalation process Shubik [1971] analyses in his paper- such that each player systematically outbids the opponent by 5 cents, till one player stops; if so, the player who stops loses his last bid, and the opponent, who gets the dollar, just pays 5 cents more than the loser: so he (almost) pays the second-price, that is to say the game looks like a second-price all-pay auction.

To give a more economic (political) example, consider an electoral campaign that leads a candidate to visiting a given town several times in order to persuade the inhabitants of the city to vote for him. Suppose that the citizens vote for the candidate that most visited their city. At first sight, this game is a first-price all-pay auction, given that each candidate loses the money and the time he invests in each visit. Yet it is also close to a war of attrition

\footnotetext{
h Shubik's game is in fact neither exactly a first-price all-pay auction nor a second price all-pay auction. It is more complex. There are potentially more than two bidders (a large crowd around the auctioneer) and only the two highest bidders pay their bid. The way ties are resolved is quite original, given that the dollar goes to the bidder closest to the auctioneer (so, at the start of the game, especially if the crowd forms a disk around the auctioneer, a potential bidder does not know if he will be the lucky closest person). Shubik expects an escalation process that lasts a long time, players overbidding each other by 5 cents, but he is not even sure that the game will start. And Shubik tells us nothing about what happens if nobody makes a bid. Does the auctioneer keep the dollar? If so, there is a discontinuity between what happens for no bid (i.e. a bid of 0 cents) and what happens for a single bid of the lowest amount ( 5 cents). For the game and the escalation process to start, Shubik expects a kind of bounded rationality in that he hopes that 'the propensity to calculate does not settle in until at least two bids have been made'.... This easily explains why Shubik's game is more than an all-pay auction and that it has been studied in many different ways.
} 
because the visits are sequential. So suppose that, at a given time $t$, both candidates visited the town a same number of times. But assume that one of the candidates, for possibly different reasons - he has not enough money, he prefers visiting other towns...- stops visiting the town. Then one (and only one) additional visit is enough for the opponent to be voted by the inhabitants of the city. So the winner just pays one more visit than the loser. This looks like a second-price all-pay auction. This example is linked to vote-buying literature (see for example Dekel et al. [2008]).

To summarize, when a first-price all-pay auction gives rise to a dynamic process of overbidding, then it can become close to a war of attrition, i.e. a second-price all-pay auction.

\section{Best-Reply Matching Equilibria, Generalized Best-Reply Matching Equilibria, Nash Equilibria and Payoffs}

What about payoffs? In the second-price all-pay auction, the NE always leads to a null payoff (in the continuous setting) or a small positive payoff in the discrete setting; this (slight) positivity is due to the fact that bid 0 always yields a non negative payoff, regardless of the bids chosen by the opponent.

With BRM and GBRM, we get different results.

We first come back to the BRME.

For M not too far from V, the BRME payoffs can be higher than the NE ones. For example, for $\mathrm{V}=3$ and $\mathrm{M}=5$, the $\mathrm{NE}$ payoff is $1589.5 / 293=5.425$, whereas the BRME payoff is 5.457 $(=180(6.5 \times 180+5(481-180))+36(8 \times 180+5.5 \times 36+4(481-180-36))+$ $45(8 \times 180+7 x 36+4.5 \times 45+3 x 60+3 x 80+3 \times 80)+60(8 x 180+7 x 36+6 x 45+3.5 \times 60+2 x 80+$ $2 x 80)+80(8 x 180+7 x 36+6 x 45+5 x 60+2.5 x 80+1 \times 80)+80(8 x 180+7 \times 36+6 x 45+5 x 60+$ $\left.4 x 80+1.5 x 80) / 481^{2}=1262620.5 / 481^{2}\right)$.

For $\mathrm{V}=9$ and $\mathrm{M}=12$, the BRME is 13.528 , whereas the NE payoff is only 12.477 .

For $V=30$ and $M=60$ (and bids in increments of ten) the results are reversed: the NE payoff is 64.157 and the BRME payoff is slightly lower $\left(24082745 / 613^{2}=64.089\right)$.

In the general setting (, $\mathrm{M}$ are integers and the bids are the integers from 0 to $\mathrm{M}$ ), we get the result in proposition 2 .

Proposition 2. We call Eg(i) the payoff obtained with bid $i$ in the BRME. We get: $E g(i+1)-E g(i)=V q_{i} / 2+V q_{i+1} / 2-\sum_{j=i+1}^{M} q_{j} \quad$ i from 0 to $M-1$.

$E g(i+1)-E g(i)$ is increasing in $i$, i from 1 to $M-1$.

For $M>2 V, E g(i)$ decreases from bid 1 to bid $M-V$, then increases up to $M$.

Proof see Appendix D. 
It derives from proposition 2 that the shape of the payoff function goes as follows. Often $E g(1)>E g(0)$ due to the fact that $\frac{V q_{0}}{2}+\frac{V q_{1}}{2}-\sum_{j=1}^{M} q_{j}>0$ (because of the large value of $\mathrm{q}_{0}$ ). Then, generally, the payoff function decreases (due to the fact that $\frac{V q_{1}}{2}+\frac{V q_{2}}{2}-$ $\sum_{j=2}^{M} q_{j}<0$ ) for a while; and, when $E g(i+1)-E g(i)$ becomes positive, the payoff function increases up to bid $M$. We represent the net payoff function for $V=9$ and $M=12$ in Fig. 6 .

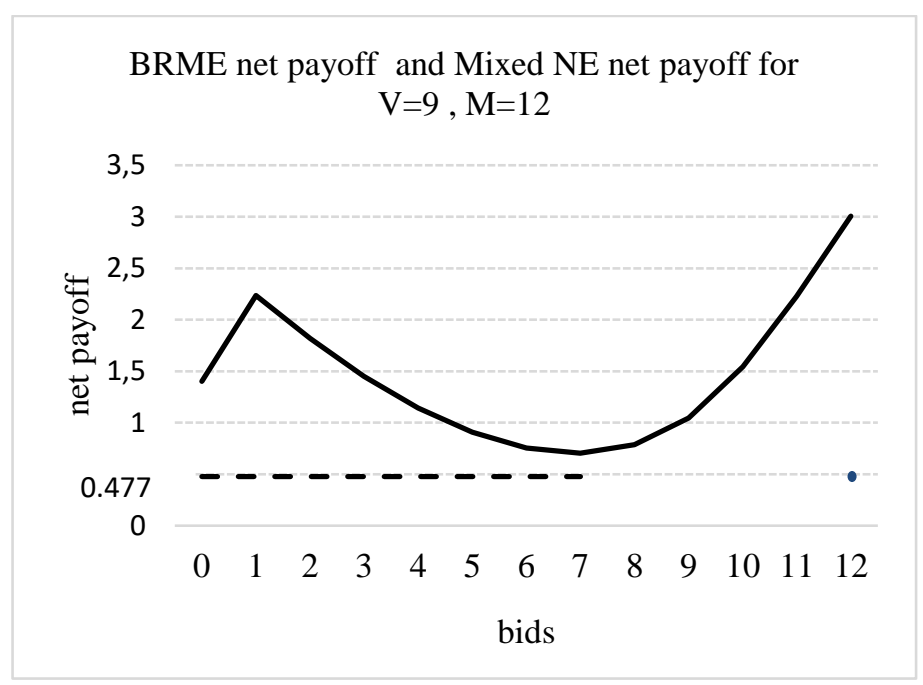

Fig. 6. BRME net payoffs (full curve) and mixed NE net payoffs (dashed line).

We comment on these payoffs. If we compare the payoffs in Fig. 6 and the probabilities assigned to the bids in Fig. 2c, we may feel uncomfortable in that the evolution of the payoffs does not follow the evolution of the probabilities. For example, $\mathrm{q}_{1}$ is the smallest probability but bid 1 yields one of the highest net payoffs; and $\mathrm{q}_{\mathrm{i}}$ increases from 1 to 7 whereas the net payoffs decrease at the same time. Yet the player, regardless of his chosen bid, never gets a negative net payoff and he always gets a net payoff larger than the NE one: his lowest net payoff, obtained for bid 7, is equal to 0.704 which is higher than 0 and higher than the NE net payoff 0.477. So the BRME is of course not stabilized as regards the Nash logic, but its own consistency ensures large payoffs.

Yet the payoffs may become negative, notably when $\mathrm{M}$ is much larger than $\mathrm{V}$. In that case $\mathrm{q}_{0}$ tends towards $1 / 2$, and all the other probabilities tend towards 0 (but are still increasing in i from 1 to $\mathrm{V}+1$ and constant from $\mathrm{V}+1$ to $\mathrm{M})$ because $\sum_{i=1}^{V} q_{i}<V q_{V}=V q_{0} /(M-V+1) \rightarrow 0$, and $\sum_{i=V+1}^{M} q_{i}=q_{0}(M-V+2)(M-V) /(M-V+1)^{2} \rightarrow q_{0}$, so q $q_{0}$ and $\sum_{i=V+1}^{M} q_{i} \rightarrow 1 / 2$.

Accordingly, when $M$ is large, the BRM probabilities are shared on bid 0 (probability $1 / 2$ ) and homogenously shared over the set of bids from $\mathrm{V}+1$ to $\mathrm{M}$ (probability $1 / 2$ on this set). We have a kind of bimodal distribution, $1 / 2$ on bid 0 and $1 / 2$ on a set (each bid in the set being played with the same probability). 
It follows that for large values of $\mathrm{M}$, a player often suffers from the winner's curse. Playing high bids leads him to often win the prize, but he pays too much given that he often wins against a player who bids too much.

Proposition 3. For large values of $M, M$ large in comparison to $V(M / V \rightarrow+\infty, V$ is a constant), the mean net loss of a player, at the BRME, is equal to $1 / 12^{\text {th }}$ of his budget $M$. The main net loss is obtained for the bid $M-V$ : the player loses $1 / 4^{\text {th }}$ of $M$.

Proof see Appendix E.

We now turn to GBRM. The degree of liberty in the way of weighting the best responses i.e. in choosing the probability distributions over the sets of best responses - gives rise to a large set of payoffs: players can get payoffs much larger than the NE payoff, but they may also get lower, negative payoffs, depending on the best responses they focus on.

Before talking about some specific payoffs, we specify the set of GBRME and their associated payoffs.

Proposition 4. For any probability distribution over each set of best responses, there exists a symmetric GBRME. These equilibria lead to a closed interval of net payoffs that includes all the payoffs in [V/2-M/4, V/4]. The maximal symmetric equilibrium net payoff will not be higher than V/2-1/4 and the minimal one will not be lower than -V/4-M/4.

Proof see Appendix F.

Given that all the net payoffs in the interval $[\mathrm{V} / 2-\mathrm{M} / 4, \mathrm{~V} / 4]$ are reachable, players can get large positive net payoffs (V/4 is far from a mixed NE null payoff) but they may also get very negative ones, notably if $M>>2$. So the first reaction may be to say that the degree of liberty allowed by the GBRME concept has the defects of its qualities. On the one hand, it allows us to give a consistent foundation to different potential observed bidding behaviors and their associated payoffs, but on the other hand, it is not very useful if used to predict the payoffs in a second-price all-pay auction game. The second reaction, which involves two steps, is more positive and is linked to behavioral economics. Firstly, true facts often reduce the acceptable probability distributions on the sets of best responses; for example, if we work with senior bidders, usually known as being cautious, we automatically weight the best responses in a given way and so we reduce the set of achievable equilibria and associated payoffs. Secondly, a large range of possible payoffs should encourage players to try to reach the largest positive payoffs, so should lead them to learning to select best responses in a way to get nice payoffs.

We now give some examples. We first observe that by selecting cautious best responses as in matrix $6 \mathrm{~b}$, which leads both players to putting the same probability $1 /(\mathrm{V}+1)$ on each bid from 0 to $\mathrm{V}$, the players get a nice positive payoff. 
Proposition 5. The GBRME that assigns the probability $1 /(V+1)$ to each bid from 0 to $V$ leads to a null net payoff in the first-price all-pay auction. But it leads to the net payoff $\left(V^{2}+2 V\right) /(6 V+6)$ in the second-price all-pay auction. Moreover, in the second-price allpay auction, the net payoff obtained with each played bid is positive.

\section{Proof see Appendix G.}

It follows from proposition 5 that by playing this GBRME in the second-price all-pay auction, the players are as safe as in the NE, given that they get a positive net payoff with each played bid. And the net payoff is much larger than the NE one, especially if $\mathrm{V}$ is large (for $\mathrm{V}=3$ and $\mathrm{M}=6$, respectively $\mathrm{V}=9$ and $\mathrm{M}=12$, the NE net payoff is 0.4157 , respectively 0.477 , whereas the GBRME net payoff is 0.625 , respectively 1.65$)$. More generally, the GBRME payoff is close to $\mathrm{V} / 6$ when $\mathrm{V}$ is large.

We now come back, in the general setting ( $\mathrm{M}, \mathrm{V}$ and the bids are integers), to our students who focus on $0, \mathrm{~V}$ and $\mathrm{M}$ (more than $4 / 5$ of the students only play these bids in Game 2); observe that for any bid from 0 to $\mathrm{M}$, at least one of these three bids is a best response to it. So we get the game in matrix $7 \mathrm{a}$, and the best-reply matrix $7 \mathrm{~b}$ ('...' represents the other bids and probabilities).

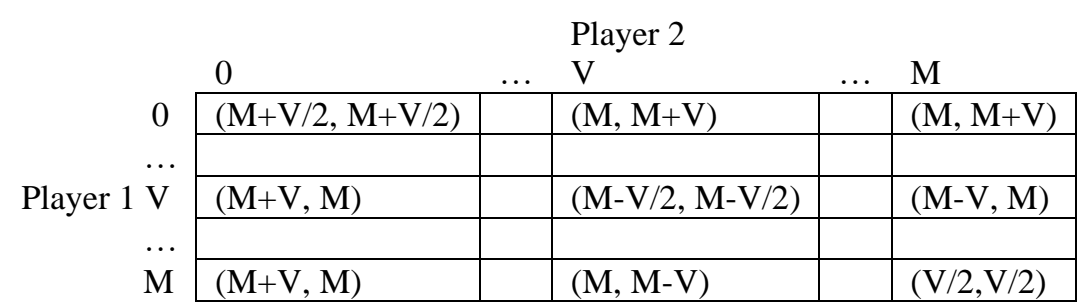

Matrix 7a. Payoffs with focal values $0, \mathrm{~V}$ and $\mathrm{M}$.

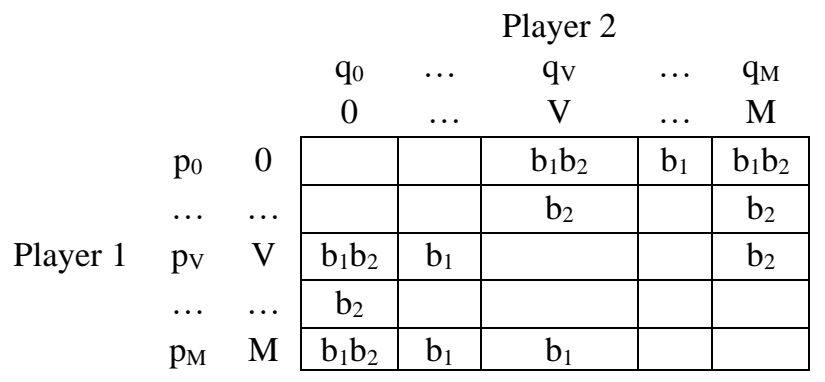

Matrix 7b. Best-reply Matrix with $0, \mathrm{~V}$ and M.

For any values of $\mathrm{V}$ and $\mathrm{M}$, given that $\mathrm{p}_{\mathrm{i}}$ and $\mathrm{q}_{\mathrm{i}}, \mathrm{i}$ from 1 to $\mathrm{M}-1, i \neq V$, are equal to 0 (the associated bids being never chosen as best responses), the GBRM equations become:
$p_{0}=q_{V} / 2+q_{M}$
$q_{0}=p_{V} / 2+p_{M}$
$p_{V}=q_{0} / 2$
$q_{V}=p_{0} / 2$
$p_{M}=q_{0} / 2+q_{V} / 2$
$q_{M}=p_{0} / 2+p_{V} / 2$ 
$p_{0}+p_{V}+p_{M}=1 \quad q_{0}+q_{V}+q_{M}=1$.

The unique solution of this system of equations is $p_{0}=q_{0}=4 / 9, p_{V}=q_{V}=2 / 9, p_{M}=q_{M}=3 / 9$.

These probabilities exploit true facts: bid 0 is played more than $\mathrm{M}$ because it is the unique best response to $\mathrm{M}$ and is also one among the 2 best responses to $\mathrm{V}$. $\mathrm{M}$ is more played than $\mathrm{V}$ because each time $\mathrm{V}$ is a best response, $\mathrm{M}$ is a best response too, and $\mathrm{M}$ is also a best response to $\mathrm{V}$. These facts may easily be observed by a real player, so he can play in accordance with the hierarchy $p_{0}>p_{M}>p_{V}$ (like the students in Game 2).

An important fact is that the probabilities do not depend on the values of $\mathrm{V}$ and $\mathrm{M}$ (what matters is that $M+V>M+V / 2, M>M-V / 2, M>V / 2$ and $M>M-V$, which is true for all values of $\mathrm{M}$ and $\mathrm{V}$, given that $M \geq V$ ). This derives from the fact that, contrary to the mixed NE concept, BRME and GBRME are ordinal concepts, so only take into account the sign of the differences in payoffs. Yet this may imply bad payoffs. As a matter of fact, the net payoff is equal to: $4 / 9 x 4 \mathrm{~V} / 18+2 / 9(4 \mathrm{~V} / 9-\mathrm{V} / 9-3 \mathrm{~V} / 9)+3 / 9(4 \mathrm{~V} / 9+3(\mathrm{~V} / 2-\mathrm{M}) / 9)=24.5 \mathrm{~V} / 81$ $M / 9$. It follows that the net payoff is negative as soon as $M>24.5 \mathrm{~V} / 9$.

Our students do not lose money even if their net payoff is barely positive (their payoff is $60.026>60$ ), because $\mathrm{M} / \mathrm{V}$ is not too large: in the experiment, $M=2 V<24.5 V / 9$. So it is possible that the students (mainly) focus on $0, V=30$ and $M=60$, because they estimate that the possible loss with $\mathrm{M}$ is not large enough to prevent them from bidding $\mathrm{M}$. In other terms, $\mathrm{M}$ is not felt as being risky. We can reasonably conjecture that the students would behave differently for other values of $\mathrm{V}, \mathrm{M}$, and the ratio $\mathrm{M} / \mathrm{V}^{\mathrm{i}}$. There may exist a kind of bifurcation in the focal bids chosen as best replies when the values of $\mathrm{M}, \mathrm{V}$ and $\mathrm{M} / \mathrm{V}$ change and exceed threshold values.

An extreme way of playing, notably useful when M/V becomes large, allowed by GBRM, consists in selecting only the focal bids 0 and $\mathrm{V}$ as best responses; this is possible because 0 or $\mathrm{V}$ belong to the best responses to each possible bid ( 0 is a best response to all bids higher than or equal to $\mathrm{V}$, and $\mathrm{V}$ is a best response to each bid lower than $\mathrm{V}$ ). In that case, the GBRM equations, after deleting all the null probabilities, reduce to $q_{0}=p_{V}$ and $q_{V}=p_{0}$, each player bidding 0 as often as the opponent bids $\mathrm{V}$ and vice versa. The symmetric GBRME, which consists in bidding 0 and $\mathrm{V}$ with probability $1 / 2$, leads to the large net positive payoff $\mathrm{V} / 4$, which is much larger than the mixed NE net payoff when $\mathrm{V}$ is large (for $\mathrm{V}=9$ and $\mathrm{M}=12, \mathrm{~V} / 4=2.25$ whereas the mixed NE net payoff is only 0.477 ).

In other terms, if real players behave in accordance with GBRM, then it is crucial to know what is focal for a player, and if a change in the values of $\mathrm{V}$ and $\mathrm{M}$ is able to induce an appropriate change in the focal bids and in the way of selecting best responses, in order always to get nice positive payoffs.

\footnotetext{
${ }^{\mathrm{i}}$ Other experiments with other students give support to this conjecture. The percentage of players playing $\mathrm{M}$ shrinks when $\mathrm{M}$ becomes much larger than $\mathrm{V}$, and new focal values emerge, like $2 \mathrm{~V}$.
} 


\section{Concluding Remarks}

We have shown in this paper that real behavior, in second-price all-pay auctions, fits much better with generalized best-reply matching than with mixed Nash equilibria. Second-price all-pay auctions are games with many best responses to a given strategy. In such games, players can adopt a best-reply matching behavior and combine it with additional characteristics, like cautiousness for example. Depending on the way players select their best responses, the equilibrium behavior and payoffs will be different.

Whereas the mixed Nash equilibrium behavior always leads to a small positive payoff (in the discrete setting) or null payoff (in the continuous setting), a best-reply (and a generalized best-reply) matching behavior can lead to lose or to get a lot of money.

With best-reply matching, when $\mathrm{M}$ is close to $\mathrm{V}$, players get a nice payoff, but when $\mathrm{M}$ is far from $\mathrm{V}$, the bidders lose money. With generalized best-reply matching, all depends on the way players choose among their best responses. Some best responses may be more focal, hence be more chosen than others, like $0, \mathrm{~V}$ and $\mathrm{M}$. In that case, the payoff is positive when $\mathrm{M} / \mathrm{V}$ is lower than a given threshold, negative otherwise. When players choose cautious bids in the best response set, then they usually make money. For example, when the bidders focus on the lowest possible best responses, they get a net payoff close to V/6 (when $\mathrm{V}$ is large). And if they only focus on 0 or $\mathrm{V}$ as best responses, each bidder gets a net payoff $\mathrm{V} / 4$, even if $\mathrm{M} / \mathrm{V}$ is large.

$\mathrm{M}$ seems to be a dangerous focal point, when it becomes large relative to $\mathrm{V}$, because bidding $\mathrm{M}$ when the opponent also bids $\mathrm{M}$ leads to losing a lot of money. Yet other experiments with other students not reproduced in this paper highlight a more subtle fact. When $\mathrm{M}$ is large in comparison to $\mathrm{V}$ (for example $M \geq 3 \mathrm{~V}$ ) students focus less on $\mathrm{M}$. As a consequence, $\mathrm{M}$ stops acting as a bad focal point. Students prefer focusing on $0, \mathrm{~V}$ and $2 \mathrm{~V}$ for example. So the negative payoffs the bidders can get by playing $\mathrm{M}$ too often, may be observed more when $\mathrm{M}$ is larger than $2 \mathrm{~V}$ but not too large. This raises the question of where $\mathrm{M}$ comes from, and, as a consequence, the question of the real number of players in the game.

For example, when the second-price all-pay auction is a true auction, then of course there is a third player, the auctioneer, who takes the bids and offers the prize, and the true game can be viewed as a three-player zero-sum game. In that case, in the mixed Nash equilibrium, the payoffs of the auctioneer and the two bidders are slightly negative and positive (are equal to 0 in the continuous setting). With generalized best-reply matching, the auctioneer and the bidders may alternately be losers and winners, depending on the value of $\mathrm{M} / \mathrm{V}$ and on the best responses the bidders focus on. Observe also that $\mathrm{M}$ can be the budget of the bidders, but it may also be arbitrarily fixed by the rules of the auction. We may also view the second-price all-pay auction as a "casino" game, like Shubik's [1971] dollar auction game; in this game, there is an auctioneer and he can, if he wants, set an upper bound $\mathrm{M}$ to invest in the game (if so, he surely chooses the amount that leads to the largest bids). But he may also fix no upper bound (M does not exist). Shubik [1971] observed that, with no upper bound, the game is usually highly beneficial to the auctioneer, 
in that it is not uncommon for him to get a total payoff between 3 and 5 dollars. To avoid such a situation, in a casino game, a game authority regulation could impose the value of $\mathrm{M}$, so that the players, even when focusing on $\mathrm{M}$ (and 0 and $\mathrm{V}$ ), do not lose money or at least not too much.

But what happens in real life? A seller rarely uses a second-price all-pay auction when he wants to sell an object, so second-price all-pay auctions are seldom true auctions. Usually, a second-price all-pay auction is used to study an economic war of attrition.

For example, consider the theory of exit from a duopoly (see for example Fudenberg and Tirole [1986]), where two firms incur a loss by staying in the duopoly, but where the remaining firm (if the other firm leaves the market) gets a positive monopoly payoff. In this game, $\mathrm{V}$ is the monopoly payoff, the bids are the losses incurred each period by staying in the market, and $\mathrm{M}$ is the maximal total loss a firm can incur before being constrained to cease trading. $\mathrm{M}$ is partly fixed by law, so could be changed; in France for example, a firm has to declare the state of insolvency at most 45 days after having observed it, so $\mathrm{M}$ is linked to these 45 days. Observe also that, in addition to the law player, there is an additional player, who would like a more generous deadline (so that $\mathrm{M}$ is larger): the consumer, who prefers the duopoly to the monopoly, because he pays less for a same amount.

More generally, there are many economic or political contexts where $\mathrm{M}$ is arbitrarily fixed and could be changed. Consider, as a last example, the presidential electoral process in France. For reasons of equality, there are campaign spending limits. In 2017 (the limit amount $\mathrm{M}$ can change from one campaign to the other), each candidate could at most invest 16.851 Million euros if eliminated in the first round of the presidential electoral process, 22.509 Million euros if he reached the second round. These amounts are fixed by law. So, if we assume that the result of the electoral process is positively linked to the amount invested in the campaign, we get a war of attrition ${ }^{\mathrm{j}}$ with an arbitrary limit $\mathrm{M}, \mathrm{V}$ being the value of winning the election. And one may wonder if changing M, or even deleting M, may change the electoral campaign and its result (see for example Che and Gale [1998], Pastine and Pastine [2012]).

To conclude, $\mathrm{M}$, in economic or political second-price all-pay auctions, when it exists, is not always a natural limit. Given that the value of the ratio M/V may have an impact on the focal values of the bidders, and hence on their behavior and payoff, this is not a neutral observation.

\section{Acknowledgments}

I wish to thank two referees for helpful suggestions and comments and I also thank the L3 students (year's class 2014/2015 and year's class 2016/2017) at the Faculté des Sciences

j This game can be seen as a first-price or a second-price all-pay auction, depending on the way the candidates are able to adapt their spending to that of the others. 
Economiques et de Gestion (Faculty of Economic and Management Sciences) of the University of Strasbourg who played the games with an endless supply of good humor.

\section{Appendix A. Proof of result 1 (Umbhauer [2017])}

Consider player 1 . If $V=1$, there is no dominated strategy. If $V>1$, the bids from $\mathrm{M}-\mathrm{V} / 2+1 / 2$ to $\mathrm{M}-1$ are weakly dominated by $\mathrm{M}$ and played with probability 0 .

$\mathrm{M}-\mathrm{V} / 2-1 / 2$ and $\mathrm{M}$ lead to the same payoff except if player 2 bids $\mathrm{M}-\mathrm{V} / 2-1 / 2$ or $\mathrm{M}$. So player 1 gets the same payoff with both bids if and only if:

$q_{M-V / 2-1 / 2}(V+1 / 2)+q_{M}(V / 2+1 / 2)=q_{M-V / 2-1 / 2}(3 V / 2+1 / 2)+q_{M}(V / 2)$

We get $\quad q_{M-V / 2-1 / 2}=q_{M} / V$.

We now compare the bids $\mathrm{M}-\mathrm{V} / 2-3 / 2$ and $\mathrm{M}-\mathrm{V} / 2-1 / 2$. Both lead to the same payoff, except if player 2 bids $\mathrm{M}-\mathrm{V} / 2-3 / 2, \mathrm{M}-\mathrm{V} / 2-1 / 2$ or $\mathrm{M}$. We need:

$q_{M-V / 2-3 / 2}(V+3 / 2)+q_{M-V / 2-1 / 2}(V / 2+3 / 2)+q_{M}(V / 2+3 / 2)=$

$q_{M-V / 2-3 / 2}(3 V / 2+3 / 2)+q_{M-V / 2-1 / 2}(V+1 / 2)+q_{M}(V / 2+1 / 2)$

hence $q_{M-V / 2-3 / 2}(-V / 2)+q_{M-V / 2-1 / 2}(-V / 2+1)+q_{M}=0$.

We get: $q_{M-V / 2-3 / 2}=q_{M}\left(1 / \mathrm{V}+2 / V^{2}\right)$.

More generally $M-V / 2-1 / 2-j$ and $M-V / 2-1 / 2-j-1$, j from 1 to $M-V / 2-3 / 2$, lead to the same payoff, except if player 2 bids $M-V / 2-1 / 2-k$ or $M$, with $k$ going from 0 to $j+1$.

We need:

$q_{M-\frac{V}{2}-\frac{1}{2}-j-1}\left(V+\frac{3}{2}+j\right)+q_{M-\frac{V}{2}-\frac{1}{2}-j}\left(\frac{V}{2}+\frac{3}{2}+j\right)+\sum_{k=0}^{j-1} q_{M-\frac{V}{2}-\frac{1}{2}-k}\left(\frac{V}{2}+\frac{3}{2}+j\right)+q_{M}\left(\frac{V}{2}+\frac{3}{2}+j\right)=$

$q_{M-\frac{V}{2}-\frac{1}{2}-j-1}\left(\frac{3 V}{2}+\frac{3}{2}+j\right)+q_{M-\frac{V}{2}-\frac{1}{2}-j}\left(V+\frac{1}{2}+j\right)+\sum_{k=0}^{j-1} q_{M-\frac{V}{2}-\frac{1}{2}-k}\left(\frac{V}{2}+\frac{1}{2}+j\right)+q_{M}\left(\frac{V}{2}+\frac{1}{2}+j\right)$.

Hence $q_{M-\frac{V}{2}-\frac{3}{2}-j}\left(\frac{-V}{2}\right)+q_{M-\frac{V}{2}-\frac{1}{2}-j}\left(\frac{-V}{2}+1\right)+\sum_{k=0}^{j-1} q_{M-\frac{V}{2}-\frac{1}{2}-k}+q_{M}=0$.

We also get:

$$
q_{M-\frac{V}{2}-\frac{3}{2}-(j+1)}\left(\frac{-V}{2}\right)+q_{M-\frac{V}{2}-\frac{1}{2}-(j+1)}\left(\frac{-V}{2}+1\right)+q_{M-\frac{V}{2}-\frac{1}{2}-j}+\sum_{k=0}^{j-1} q_{M-\frac{V}{2}-\frac{1}{2}-k}+q_{M}=0 .
$$

It follows $q_{M-V / 2-j-5 / 2}=2 q_{M-V / 2-j-3 / 2} / V+q_{M-V / 2-j-1 / 2}$ for any $\mathrm{j}$ from 0 to $\mathrm{M}-\mathrm{V} / 2-5 / 2$.

i.e. $q_{i}=2 q_{i+1} / V+q_{i+2}$ for $\mathrm{i}$ from 0 to $\mathrm{M}-\mathrm{V} / 2-5 / 2$.

Observe that if $M=(V+1) / 2$, which requires $V=M=1$ (because $V \leq M$ ), then the proof stops with $q_{M-V / 2-1 / 2}=q_{0}=q_{M} / V=q_{1}=1 / 2$. And if $M=(V+3) / 2$, which requires $M \leq 3$, then the proof stops with $q_{1}=q_{M} / V$ and $q_{0}=q_{M}\left(1 / V+2 / V^{2}\right) . \quad M=(V+3) / 2$ implies $M=3$ and $V=3$ (and $q_{0}=5 / 17, q_{1}=3 / 17$ and $q_{3}=9 / 17$ ), or $M=2$ and $V=1$ ( and $q_{0}=3 / 5, q_{1}=q_{2}=1 / 5$ ).

The analysis is the same for player 2 , so leads to the same equilibrium probabilities for player 1 .

\section{Appendix B. Proof of result 2}

A first proof of this (almost folk) result goes back to Hendricks et al. [1988]. The following proof is from Umbhauer [2016]. 
All bids from $\mathrm{M}-\mathrm{V} / 2$ to $\mathrm{M}$ (excluded) are weakly dominated by $\mathrm{M}$, so it is conjectured that the NE strategy is a density function $\mathrm{f}($.) on $[0, \mathrm{M}-\mathrm{V} / 2]$ with a mass point on $\mathrm{M}$.

Call $\mathrm{f}_{2}($.$) player 2's equilibrium strategy. Suppose that player 1$ plays b. She wins the auction each time player 2 bids less than $b$. Her payoff $\mathrm{Eg}(\mathrm{b})$ is equal to:

$$
E g(b)=M+\int_{0}^{b}(V-x) f_{2}(x) d x-b\left(\int_{b}^{M-V / 2} f_{2}(x) d x+f_{2}(M)\right)
$$

$\mathrm{Eg}(\mathrm{b})$ has to be constant for each $\mathrm{b}$ in $[0, \mathrm{M}-\mathrm{V} / 2] \cup\{\mathrm{M}\}$.

We get $E g(M)=M+\int_{0}^{M-V / 2}(V-x) f_{2}(x) d x+\left(\frac{V}{2}-M\right) f_{2}(M)=E g\left(M-\frac{V}{2}\right)$.

We need $E g^{\prime}(b)=0$ for b in $[0, \mathrm{M}-\mathrm{V} / 2]$.

We get $(V-b) f_{2}(b)-F_{2}(M-V / 2)+F_{2}(b)-f_{2}(M)+b f_{2}(b)=0$

where $\mathrm{F}_{2}($.$) is the cumulative distribution of the density function \mathrm{f}_{2}($.$) .$

By construction $f_{2}(M)=1-F_{2}(M-V / 2)$, and the differential equation becomes

$V f_{2}(b)-1+F_{2}(b)=0$.

The solution is $F_{2}(b)=1+K e^{-b / V}$ where $\mathrm{K}$ is a constant determined as follows:

$F_{2}(0)=0$ because there is no mass point on 0 , so $K=-1$.

It follows $\mathrm{F}_{2}(\mathrm{~b})=1-\mathrm{e}^{-\mathrm{b} / \mathrm{V}}$ for $\mathrm{b}$ in $[0, \mathrm{M}-\mathrm{V} / 2], f_{2}(M)=1-F_{2}(M-V / 2)=e^{1 / 2-M / V}(<1)$, $f_{2}(b)=e^{-b / V} / V$ for $\mathrm{b}$ in $[0, \mathrm{M}-\mathrm{V} / 2]$ (and $f_{2}(b)=0$ for $\mathrm{b}$ in $] \mathrm{M}-\mathrm{V} / 2, \mathrm{M}[$ ).

By symmetry, we get $f_{l}(b)=e^{-b / V} / V$ for b in $[0, \mathrm{M}-\mathrm{V} / 2], f_{l}(M)=e^{1 / 2-M / V}$ (and $f_{l}(b)=0$ for $\mathrm{b}$ in $] \mathrm{M}-\mathrm{V} / 2, \mathrm{M}[\mathrm{)}$.

Given that $\operatorname{Eg}(0)$ is equal to $\mathrm{M}$, given that bid 0 is played at equilibrium, and given that a player gets the same payoff with each played bid, each player gets the payoff $M$, hence a net payoff equal to 0 at equilibrium.

\section{Appendix C. Proof of proposition 1}

Each bid $\mathrm{i}, \mathrm{i}$ from 1 to $\mathrm{V}$ is a best reply to all bids $\mathrm{j}, \mathrm{j}$ from 0 to $\mathrm{i}-1$.

Each bid $\mathrm{i}$, i from $\mathrm{V}+1$ to $\mathrm{M}$ is a best reply to all bids $\mathrm{j}, \mathrm{j}$ from 0 to $\mathrm{V}$ (if $M>V$ ).

Bid 0 is a best reply to $\mathrm{V}$ and the only best reply to bid $\mathrm{j}, \mathrm{j}$ from $\mathrm{V}+1$ to $\mathrm{M}$, if $M>V$, and bid 0 is the only best reply to $\mathrm{V}$ if $\mathrm{M}=\mathrm{V}$.

We get:

$p_{0}=\frac{q_{V}}{M-V+1}+\sum_{i=1}^{M-V} q_{V+i} \quad q_{0}=\frac{p_{V}}{M-V+1}+\sum_{i=1}^{M-V} p_{V+i}$.

These two equations reduce to $p_{0}=q_{V}$ and $q_{0}=p_{V}$ if $M=V$.

$$
\begin{aligned}
& p_{1}=q_{0} / M \quad q_{1}=p_{0} / M \\
& p_{2}=q_{0} / M+q_{1} /(M-1) \quad(\text { if } V>1) \quad q_{2}=p_{0} / M+p_{1} /(M-1) \quad(\text { if } V>1) \\
& p_{i}=\sum_{j=0}^{V-1} \frac{q_{j}}{M-j}+q_{V} /(M-V+1) \quad \text { i from } \mathrm{V}+1 \text { to } \mathrm{M} \text {, } \\
& q_{i}=\sum_{j=0}^{V-1} \frac{p_{j}}{M-j}+p_{V} /(M-V+1) \quad \text { i from } \mathrm{V}+1 \text { to } \mathrm{M} \text {, } \\
& \text { and } \sum_{i=0}^{M} p_{i}=\sum_{i=0}^{M} q_{i}=1 \text {. }
\end{aligned}
$$$$
p_{i}=\sum_{j=0}^{i-1} q_{j} /(M-j) \quad \text { i from } 1 \text { to } \mathrm{V} \quad q_{i}=\sum_{j=0}^{i-1} p_{j} /(M-j) \quad \text { i from } 1 \text { to } \mathrm{V}
$$

It can easily be checked that the equations defining $\mathrm{p}_{0}$ and $\mathrm{q}_{0}$ are redundant. 
We first look for a symmetric BRME.

We start with $M>V$.

We get:

$q_{0}=\frac{q_{V}}{M-V+1}+\sum_{i=1}^{M-V} q_{V+i}$

$q_{1}=q_{0} / M$

$q_{2}=q_{0} / M+q_{1} /(M-1) \quad$ (if $V>1$ )

$q_{i}=\sum_{j=0}^{i-1} q_{j} /(M-j) \quad$ i from 1 to $\mathrm{V}$

$q_{i}=\sum_{j=0}^{V-1} \frac{q_{j}}{M-j}+q_{V} /(M-V+1)$ i from $\mathrm{V}+1$ to $\mathrm{M}$.

We observe that $\sum_{i=V+1}^{M} q_{i}<q_{0}<\sum_{i=V}^{M} q_{i}$.

Suppose V>1. Given that $q_{1}=q_{0} / M$, we get $q_{2}=q_{0} / M+q_{0} /((M-1) M)=q_{0} /(M-1)$.

$q_{i}=q_{0} /(M-i+1)$ implies $q_{i+1}=q_{i}+q_{i} /(M-i)=q_{0} /(M-i+1)+q_{0} /((M-i+1)(M-i))=q_{0} /(M-i)$.

It derives by induction that $q_{i}=q_{0} /(M-i+1)$, for i from 1 to $\mathrm{V}$.

And $q_{i}=q_{0} /(M-V+1)+q_{V} /(M-V+1)=q_{0} /(M-V+1)+q_{0} /(M-V+1)^{2}=q_{0}(M-V+2) /(M-V+1)^{2}$ for i from $\mathrm{V}+1$ to $\mathrm{M}$.

It follows: $q_{0}+q_{0} / M+q_{0} /(M-1)+\ldots+q_{0} /(M-V+1)+q_{0}-q_{0} /(M-V+1)^{2}=1$.

So $q_{0}\left(2+\sum_{i=0}^{V-1} 1 /(M-i)-1 /(M-V+1)^{2}=1\right.$.

It can be checked that the result is the same for $V=1$.

We now suppose that $M=V$.

In that case the bids from $\mathrm{V}+1$ to $\mathrm{M}$ vanish and we only focus on the boxed equations (C.1) and (C.2) (and the redundant equations $p_{0}=q_{V}$ and $\left.q_{0}=p_{V}\right)$. It easily follows that $q_{0} /(1+$ $\left.\sum_{i=0}^{V-1} 1 /(M-i)\right)=1$.

Given that $1+\sum_{i=0}^{V-1} \frac{1}{M-i}=2+\sum_{i=0}^{V-1} 1 /(M-i)-1 /(M-V+1)^{2}$ (for $M=V$ ), we keep the same notation for $\mathrm{q}_{0}$ for $M>V$ and $M=V$.

It can be checked that the result also holds for $M=V=1$. It reduces to $q_{0}=q_{1}=1 / 2$.

We now show that the equilibrium is unique, by showing that a BRME is necessarily symmetric (for $M \neq 1$ ).

We observe that $q_{0}=p_{0}$ implies $q_{i}=p_{i}$ for $\mathrm{i}$ from 1 to $\mathrm{M}$.

Now we set $p_{o}=a q_{0}$ where $a$ is a constant, and we show that $a=1$.

We start with $M>V$.

We have:

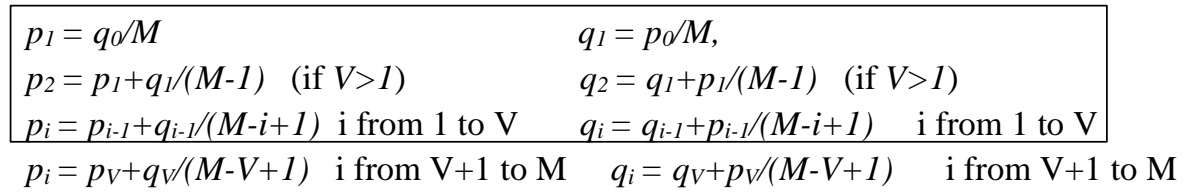




$$
p_{0}=\frac{q_{V}}{M-V+1}+\sum_{i=1}^{M-V} q_{V+i} \quad q_{0}=\frac{p_{V}}{M-V+1}+\sum_{i=1}^{M-V} p_{V+i}
$$

Suppose $V>1$.

It follows:

$$
\begin{aligned}
& p_{1}-q_{1}=\left(q_{0}-p_{0}\right) / M=(1-a) q_{0} / M \quad \text { i.e. } \quad p_{1}=q_{1}+(1-a) q_{0} / M \\
& p_{2}-q_{2}=\left(p_{1}-q_{1}\right)(1-1 /(M-1))=(1-a)(M-2) q_{0} /[M(M-1)] \\
& p_{i-} q_{i}=\left(p_{i-1}-q_{i-1}\right)(1-1 /(M-i+1))=(1-a)(M-i) q_{0} /[M(M-1)] \quad \text { i from } 2 \text { to } \mathrm{V} \\
& p_{V+i^{-}} q_{V+i}=\left(p_{V}-q_{V}\right)(1-1 /(M-V+1))=(1-a)(M-V)^{2} q_{0} /[M(M-1)(M-V+1)] \text { i from } 1 \text { to M-V. } \\
& p_{0}=\frac{q_{V}}{M-V+1}+\sum_{i=1}^{M-V} q_{V+i} \Rightarrow \quad a q_{0}=q_{V} /(M-V+1)+(M-V) q_{V+1} \\
& q_{0}=\frac{p_{V}}{M-V+1}+\sum_{i=1}^{M-V} p_{V+i} \Rightarrow \\
& q_{0}=\left(q_{V}+(1-a)(M-V) q_{0} /[M(M-1)]\right) /(M-V+1)+ \\
& (M-V)\left(q_{V+1}+(1-a)(M-V)^{2} q_{0} /[M(M-1)(M-V+1)]\right) \text {. } \\
& q_{0}=q_{V} /(M-V+1)+(M-V) q_{V+1}+(1-a) q_{0}\left((M-V)+(M-V)^{3}\right) /[M(M-1)(M-V+1)] \text {. } \\
& \text { It follows from Eq. (C.5) and (C.6): } \\
& a q_{0}=q_{0}-(1-a) q_{0}\left((M-V)+(M-V)^{3}\right) /[M(M-1)(M-V+1)] \\
& \text { i.e. }(1-a)\left(1-\left((M-V)+(M-V)^{3}\right) /[M(M-1)(M-V+1)]\right)=0 \\
& \text { i.e. } a=1 \text { except if }\left((M-V)+(M-V)^{3}\right) /[M(M-1)(M-V+1)]=1 \text {, which is not possible for } M>V \text {. } \\
& \text { By developing }\left((M-V)+(M-V)^{3}\right) /[M(M-1)(M-V+1)]-1 \text { we get the expression } 3 M V^{2}-2 M^{2} V \text { - } \\
& V^{3}+2 M-V M-V=V(V-M)(2 M-V)+M(2-V)-V \text { which is always negative. }
\end{aligned}
$$

Hence $a=1$, and the symmetric BRME is the only BRME of the game.

Easier calculations lead to the same result for $V=1$.

We now switch to $M=V$.

If $V=M=1$, it is obvious to get the infinite set of BRME, defined by: $p_{1}=q_{0}, q_{0}+q_{1}=1$, $p_{0}+p_{1}=1$.

Suppose $M>1$; given that $V=M$, we only focus on the boxed equations (C.3) and (C.4) and the equations $p_{0}=q_{V}$ and $q_{0}=p_{V}$. Given the expression of $p_{i}-q_{i}$, for $i$ from 1 to $\mathrm{V}$, we have $p_{V}=q_{V}$. Given that $p_{0}=q_{V}$ and $q_{0}=p_{V}$, we get $p_{0}=q_{0}$. So the symmetric BRME is the only BRME of the game.

\section{Appendix D. Proof of proposition 2}

We have $E g(0)=M+q_{0} V / 2$

$E g(1)=M+q_{0} V+q_{1}(V / 2-1)-1\left(1-q_{0}-q_{1}\right)=M+q_{0} V+q_{1} V / 2-1\left(1-q_{0}\right)$

$E g(i)=M+\sum_{j=0}^{i-1}(V-j) q_{j}+q_{i} V / 2-i\left(1-\sum_{j=0}^{i-1} q_{j}\right)$ i from 1 to $\mathrm{M}$.

It follows: $E g(i+1)-E g(i)=(V-i) q_{i}+\frac{q_{i+1} V}{2}-\frac{q_{i} V}{2}+i q_{i}-\left(1-\sum_{j=0}^{i} q_{j}\right)$

$=\frac{V q_{i}}{2}+\frac{V q_{i+1}}{2}-\sum_{j=i+1}^{M} q_{j}$.

And $E g(i+2)-E g(i+1)=\frac{V q_{i+1}}{2}+\frac{V q_{i+2}}{2}-\sum_{j=i+2}^{M} q_{j}$. 
Given that $q_{i+2} \geq q_{i}$ for $i$ from 1 to $\mathrm{M}-2$, and given that $-\sum_{j=i+2}^{M} q_{j}>-\sum_{j=i+1}^{M} q_{j}$ for $\mathrm{i}$ from 0 to $\mathrm{M}$-2, we get $E g(i+2)-E g(i+1)>E g(i+1)-E g(i)$ for i from 1 to $\mathrm{M}-2$. So $E g(i+1)-E g(i)$ is increasing in $\mathrm{i}$, for $\mathrm{i}$ from 1 to $\mathrm{M}-1$.

We now suppose $M>2 V$.

We get, for i from 1 to $\mathrm{M}-\mathrm{V}-1$,

$$
\begin{aligned}
& E g(V+i+1)-E g(V+i)=\frac{V q_{V+i}}{2}+\frac{V q_{V+i+1}}{2}-\sum_{j=i+1}^{M-V} q_{V+j} \\
& =q_{0}(M-V+2)(2 V+i-M) /(M-V+1)^{2}
\end{aligned}
$$

given that $q_{V+j}=q_{0}(M-V+2) /(M-V+1)^{2}$ for $\mathrm{i}$ from 1 to $\mathrm{M}-\mathrm{V}$. It follows that $E g(V+i+1)$ $E g(V+i)$ becomes positive only for $i>M-2 V$.

Hence $E g(j+1)-E g(j)$ becomes positive only for $j>M-V$.

Putting the results together, it derives that, for $M>2 V, \operatorname{Eg}(\mathrm{b})$ decreases for b from 1 to $\mathrm{M}$ $\mathrm{V}$ and increases from $\mathrm{M}-\mathrm{V}+1$ to $\mathrm{M}$.

\section{Appendix E. Proof of proposition 3}

\section{First part of the proposition.}

We calculate the mean payoff for each player when $M$ is large. We know that, when $M$ is large $(M \rightarrow+\infty)$ and $\mathrm{V}$ is a constant, then $q_{0} \rightarrow 1 / 2, \sum_{i=1}^{V} q_{i} \rightarrow 0, \sum_{i=V+1}^{M} q_{i} \rightarrow 1 / 2$, so $q_{i}=$ $a=1 /(2(M-V))$ for each i from $\mathrm{V}+1$ to $\mathrm{M}$.

We look for the payoff obtained with each played bid. We omit the bids from 1 to $\mathrm{V}$, given that they lead to a payoff which is a constant that will be multiplied by a probability (of playing the bid) so close to 0 that the sum of the payoffs obtained with these bids also tends towards 0 (each $q_{i} \rightarrow 0$ and $\sum_{i=1}^{V} q_{i} \rightarrow 0$ ). For similar reasons we omit the payoff a player gets when he meets a player who plays a bid from 1 to $\mathrm{V}$. So we calculate the payoff obtained with bid 0 , the payoff obtained with bid $\mathrm{V}+\mathrm{i}$, i from 1 to $\mathrm{M}-\mathrm{V}$, and the expected net payoff.

Net payoff obtained with bid $0=q_{0} V / 2=V / 4$.

Net payoff obtained with bid $\mathrm{V}+1=$

$q_{0} V+a(V / 2-V-1)-(V+1)(M-V-1) a=q_{0} V+a(V / 2)-a(V+1)(M-V)$.

Net payoff obtained with bid V+2 $=q_{0} V-a+a(V / 2-V-2)-(V+2)(M-V-2) a=$

$q_{0} V-a+a(V / 2)-a(V+2)(M-V-1)$.

More generally, the net payoff obtained with bid $\mathrm{V}+\mathrm{i}$ is equal to:

$q_{0} V-a-\ldots .-(i-1) a+a(V / 2)-a(V+i)(M-V-i+1), \quad$ i from 2 to $\mathrm{M}-\mathrm{V}$.

So, for $\mathrm{i}$ from 1 to $\mathrm{M}-\mathrm{V}$, the net payoff obtained with $\mathrm{V}+\mathrm{i}$ is equal to:

$V / 2+a i^{2} / 2-a V(M-V+1 / 2)-i a(M-2 V+1 / 2)$

To calculate the expected net payoff, we multiply $V / 4$ by $\mathrm{q}_{0}$, the net payoff for bid $\mathrm{V}+\mathrm{i}$ by $a$, i from 1 to $\mathrm{M}-\mathrm{V}$, and we sum these payoffs. We get:

$$
\begin{aligned}
& \frac{V}{8}+\frac{a(M-V) V}{2}-a^{2}(M-V) V\left(M-V+\frac{1}{2}\right)+a \sum_{i=1}^{M-V}\left(\frac{a i^{2}}{2}-i a\left(M-2 V+\frac{1}{2}\right)\right) . \\
& a \sum_{i=1}^{M-V}\left(\frac{a i^{2}}{2}-i a\left(M-2 V+\frac{1}{2}\right)\right)=
\end{aligned}
$$


$a^{2}(M-V)(M-V+1)(2 M-2 V+1) / 12-a^{2}(M-V)(M-V+1)(M-2 V+1 / 2) / 2$, which tends towards $2 M / 48-M / 8=-M / 12$ because $a=1 /(2(M-V))$ and because $\mathrm{V}$ and the other constants are small in comparison to $\mathrm{M}$.

$V / 8+a(M-V) V / 2-a^{2}(M-V) V(M-V+1 / 2) \rightarrow V / 8+V / 4-V / 4$ because $a=1 /(2(M-V))$.

So the BRME net payoff tends towards $-\mathrm{M} / 12$.

\section{Second part of the proposition}

Given that $M \gg 2 V$, we know from proposition 2 that the lowest payoff is obtained for bid $\mathrm{M}-\mathrm{V}$, i.e. bid $\mathrm{V}+(\mathrm{M}-2 \mathrm{~V})$. This net payoff is equal to:

$V / 2+a i^{2} / 2-a V(M-V+1 / 2)-i a(M-2 V+1 / 2)$ with $i=M-2 V$ and $a=1 /(2(M-V))$.

So it is equal to : $V / 2+a(M-2 V)^{2} / 2-a V(M-V+1 / 2)-(M-2 V) a(M-2 V+1 / 2)$ which tends towards $-\mathrm{M} / 4$ (we can omit $\mathrm{V}$ and the others constants given that only $M \rightarrow+\infty$ ).

\section{Appendix F. Proof of proposition 4}

We only look for symmetric GBRME and we only study the case $M>V$ (there is no difficulty in adapting the proof to $M=V$ ). We get the equations (F.1):

$q_{0}=\delta_{0 v} q_{V}+\sum_{i=V+1}^{M} q_{i}$

$q_{i}=\sum_{j=0}^{i-1} \delta_{i j} q_{j} \quad$ i from 1 to $\mathrm{V}$

$q_{i}=\sum_{j=0}^{V} \delta_{i j} q_{j} \quad$ i from $\mathrm{V}+1$ to $\mathrm{M} \quad$ and $\quad \sum_{i=0}^{M} q_{i}=1$.

For ease of notation we will write " $\delta$ " for all "the constants/variables $\delta_{0 \mathrm{v}}$ and $\delta_{\mathrm{ij}}, \mathrm{j}$ from 0 to $\mathrm{V}$ and $\mathrm{i}$ from $\mathrm{j}+1$ to $\mathrm{M}$ ". We call $\mathrm{D}$ the set of $\delta$ that check the constraints (F.2):

$\sum_{i=j+1}^{M} \delta_{i j}=1 \quad \mathrm{j}$ from 0 to $\mathrm{V}-1$

$\delta_{0 V}+\sum_{i=V+1}^{M} \delta_{i V}=1$

$\delta_{0 V} \geq 0, \delta_{i j} \geq 0$, j from 0 to $\mathrm{V}$ and $\mathrm{i}$ from $\mathrm{j}+1$ to $\mathrm{M}$.

There is a redundant equation in (F.1):

$q_{i}=\sum_{j=0}^{i-1} \delta_{i j} q_{j}$, i from 1 to $\mathrm{V}, q_{i}=\sum_{j=0}^{V} \delta_{i j} q_{j}$, i from $\mathrm{V}+1$ to $\mathrm{M}$, and $\sum_{i=0}^{M} q_{i}=1 \Rightarrow$

$q_{0}=1-\sum_{i=1}^{M} q_{i}=1-\sum_{j=0}^{V-1} \sum_{i=j+1}^{M} \delta_{i j} q_{j}-\sum_{j=V+1}^{M} \delta_{j V} q_{V}=1-\sum_{j=0}^{V-1} q_{j}-\left(1-\delta_{0 V}\right) q_{V}$

$=\sum_{i=V}^{M} q_{i}-\left(1-\delta_{0 V}\right) q_{V}=\sum_{i=V+1}^{M} q_{i}+\delta_{0 V} q_{V}$. So the first equation is redundant and we suppress it.

The existence of a unique symmetric GBRME for all $\delta$ in D follows by construction.

$q_{1}=\delta_{10} q_{0}=a_{1} q_{0}$ with $a_{1} \geq 0$.

$q_{2}=\delta_{20} q_{0}+\delta_{21} a_{1} q_{0}=a_{2} q_{0}$ with $a_{2} \geq 0$.

Suppose that $q_{i}=a_{i} q_{0}$ with $a_{i} \geq 0$ for $i<V$.

It follows $q_{i+1}=\sum_{j=0}^{i} \delta_{(i+1) j} q_{j}=\sum_{j=0}^{i} \delta_{(i+1) j} a_{j} q_{0}=a_{i+1} q_{0}$ with $a_{i+1} \geq 0$.

So $q_{i}=a_{i} q_{0}$ with $a_{i} \geq 0 \quad$ i from 1 to $\mathrm{V}$.

And $q_{i}=\sum_{j=0}^{V} \delta_{i j} q_{j}=\sum_{j=0}^{V} \delta_{i j} a_{j} q_{0}=a_{i} q_{0} \quad a_{i} \geq 0 \quad$ i from V+1 to M. 
We get: $\sum_{i=0}^{M} q_{i}=1 \Leftrightarrow q_{0}=1 /\left(1+\sum_{i=1}^{M} a_{i}\right) \quad$ with at least one strictly positive $a_{i}, \mathrm{i}$ from 1 to $\mathrm{M}$.

So $q_{0}$ is unique, positive and lower than 1 . Given that $q_{i}=a_{i} q_{0}$ with $a_{i} \geq 0$, and given that $\sum_{i=0}^{M} q_{i}=1$, it follows that all the $\mathrm{q}_{\mathrm{i}}$, i from 1 to $\mathrm{M}$ are unique, positive or null and lower than 1 . So, for all $\delta$ in $\mathrm{D}$, there exists a unique symmetric GBRME.

More precisions can be given on $\mathrm{q}_{0}$. Given that $q_{i}=\delta_{i 0} q_{0}+b_{i}$, with $b_{i} \geq 0$, i from 1 to $\mathrm{M}$, we get $\sum_{i=1}^{M} q_{i}=\sum_{i=1}^{M}\left(\delta_{i 0} q_{0}+b_{i}\right)=q_{0}+\sum_{i=1}^{M} b_{i}$. It follows that $\sum_{i=0}^{M} q_{i}=1 \Leftrightarrow 2 q_{0}+$ $\sum_{i=1}^{M} b_{i}=1$ so $q_{0}<1 / 2$ given that each $b_{i}$ is positive or null (i from 1 to $\mathrm{M}$ ), and at least one of them is strictly positive if $V>1$ (if $V=1$, qo can be equal to $1 / 2$ ).

We now look at player 1's net payoff (the analysis is symmetric for player 2). It is equal to :

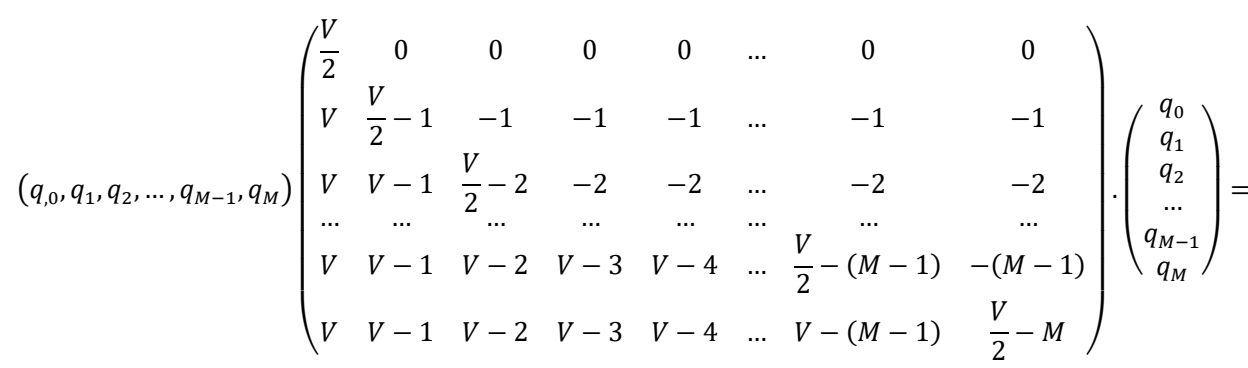

$\frac{V}{2} q_{0}^{2}+\sum_{i=1}^{M-1} q_{i}\left(\sum_{j=0}^{i-1}(V-j) q_{j}+\left(\frac{V}{2}-i\right) q_{i}-i \sum_{j=i+1}^{M} q_{j}\right)+q_{M}\left(\sum_{j=0}^{M-1}(V-j) q_{j}+\left(\frac{V}{2}-M\right) q_{M}\right)=$

$\frac{V}{2} \sum_{i=0}^{M} q_{i}^{2}+V \sum_{j=i+1}^{M} \sum_{i=0}^{M-1} q_{i} q_{j}-\sum_{i=1}^{M-1} i q_{i}\left(q_{i}+2 \sum_{j=i+1}^{M} q_{j}\right)-M q_{M}^{2}=\frac{V}{2}\left[\sum_{i=0}^{M} q_{i}\right]^{2}-$ $\sum_{i=1}^{M}\left[\sum_{j=i}^{M} q_{j}\right]^{2}=\frac{V}{2}-\sum_{i=1}^{M}\left[\sum_{j=i}^{M} q_{j}\right]^{2}$.

When $M=V$ we get the same result.

We write $g()=.\frac{V}{2}-\sum_{i=1}^{M}\left[\sum_{j=i}^{M} q_{j}\right]^{2}$ with $q_{i}=a_{i} /\left(1+\sum_{i=1}^{M} a_{i}\right)$ where $a_{i}$ is defined as above, i from 1 to $\mathrm{M}$, in function of $\delta$. So $\mathrm{g}($.$) is a function of \delta$ and can be maximized and minimized on $\delta$ subject to $\delta \in D$.

Given that $\mathrm{g}(\delta)$ is continuous on D, given that D is closed and bounded, Weierstrass's theorem ensures that $\mathrm{g}(\delta)$ has a maximum and a minimum on $\mathrm{D}$.

We give a trivial upper bound for the maximum. Given that $\left(\sum_{j=1}^{M} q_{j}\right)^{2}=\left(1-q_{0}\right)^{2}$ and given that $q_{0}<1 / 2$, we get $\frac{V}{2}-\sum_{i=1}^{M}\left[\sum_{j=i}^{M} q_{j}\right]^{2}<\frac{V}{2}-\frac{1}{4}$.

This result also holds when $M=V$.

We provide a lower bound:

Given that $q_{0} \geq \sum_{i=V+1}^{M} q_{i}$

$\frac{V}{2}-\sum_{i=1}^{M}\left[\sum_{j=i}^{M} q_{j}\right]^{2}=\frac{V}{2}-\sum_{i=1}^{V}\left[\sum_{j=i}^{M} q_{j}\right]^{2}-\sum_{i=V+1}^{M}\left[\sum_{j=i}^{M} q_{j}\right]^{2}$

$\sum_{i=V+1}^{M}\left[\sum_{j=i}^{M} q_{j}\right]^{2} \leq(M-V)\left(\sum_{i=V+1}^{M} q_{i}\right)^{2}$ 


$$
\begin{aligned}
& \sum_{i=1}^{V}\left[\sum_{j=i}^{M} q_{j}\right]^{2} \leq V\left(1-q_{0}\right)^{2} . \\
& \frac{V}{2}-\sum_{i=1}^{V}\left[\sum_{j=i}^{M} q_{j}\right]^{2}-\sum_{i=V+1}^{M}\left[\sum_{j=i}^{M} q_{j}\right]^{2} \geq \frac{V}{2}-V\left(1-q_{0}\right)^{2}-(M-V)\left(\sum_{i=V+1}^{M} q_{i}\right)^{2} \\
& \geq \frac{V}{2}-V-(M-V) q_{0}^{2} \\
& \geq \frac{V}{2}-V-(M-V) / 4 \\
& \geq-V / 4-M / 4
\end{aligned}
$$

This result also holds for $M=V$.

Given that $\left(q_{0}=1 / 2, q_{V}=1 / 2, q_{i}=0, i \neq 0, V\right)$ satisfies the equations (F.1) when $\delta_{0 V}=1$ and $\delta_{V i}=1$, $\mathrm{i}$ from 0 to $\mathrm{V}-1$, the other $\delta$ variables being equal to 0 , the maximum is necessarily higher than or equal to $V / 4$. And given that $\left(q_{0}=1 / 2, q_{M}=1 / 2, q_{i}=0, i \neq 0, M\right)$ satisfies the equations (F.1) when $\delta_{\mathrm{Mi}}=1$, i from 0 to $\mathrm{V}$, the other $\delta$ variables being equal to 0 , the minimum is necessarily lower than or equal to $V / 2-M / 4$.

$\mathrm{D}$ is convex and $\mathrm{g}(\delta)$ is continuous on $\mathrm{D}$. So the intermediate value theorem ensures that all the payoffs between the minimum and the maximum, and a fortiori, any payoff between $\mathrm{V} / 2-\mathrm{M} / 4$ and $\mathrm{V} / 4$, can be observed for some specific $\delta$. Of course, this last result is of interest only if $M>V$.

\section{Appendix G. Proof of proposition 5}

We first calculate the net payoff in the first-price all-pay auction. It is well-known that the discrete NE leads to playing each bid from 0 to $\mathrm{V}-1$ with probability $1 / \mathrm{V}$ and that the NE payoff is 0.5 (so each bid from 0 to $\mathrm{V}-1$ leads to the payoff 0.5 in the NE).

It follows that, in the GBRME:

bid 0 leads to the net payoff $0.5 \mathrm{~V} /(\mathrm{V}+1)$.

bid 1 leads to the net payoff $0.5 V /(V+1)-1 /(V+1) \quad$ (because we have to add the payoff obtained by bid 1 when confronted to bid $\mathrm{V})$.

bid i leads to the net payoff $0.5 V /(V+1)-i /(V+1) \quad$ i from 2 to $\mathrm{V}-1$.

bid $\mathrm{V}$ leads to the net payoff $-0.5 \mathrm{~V} /(\mathrm{V}+1)$.

And the GBRME net payoff is equal to:

$0.5 V^{2} /(V+1)^{2}-(1+2+\ldots+V-1) /(V+1)^{2}-0.5 V /(V+1)^{2}=\left[0.5 V^{2}-0.5 V(V-1)-0.5 V\right] /(V+1)^{2}=$ 0 .

We now focus on the second-price all-pay auction.

Bid 0 leads to the net payoff $0.5 V /(V+1)$.

Bid 1 leads to the net payoff $[V+V / 2-V] /(V+1)$.

Bid $i$ leads to the net payoff $\left[\sum_{j=0}^{i-1}(V-j)+V / 2-i(V-i+1)\right] /(V+1)$ i from 2 to $\mathrm{V}$.

We get $\left[\sum_{j=0}^{i-1}(V-j)+V / 2-i(V-i+1)\right] /(V+1)=[V / 2+i(i-1) / 2] /(V+1)$

for $i$ from 1 to $\mathrm{V}$.

And the GBRME net payoff becomes:

$\left[0.5 V(V+1)+0.5 \sum_{i=1}^{V} i^{2}-0.5 \sum_{i=1}^{V} i\right] /(V+1)^{2}=0.5[V(V+1)+V(V+1)(2 V+1) / 6-$

$0.5 V(V+1)] /(V+1)^{2}=0.5 V[0.5+(2 V+1) / 6] /(V+1)=\left(V^{2}+2 V\right) /(6(V+1))$. 
Regardless of the played bid, the player gets a positive net payoff, given that bid 0 leads to the net payoff $0.5 V /(V+1)$ and that bid i leads to the net payoff $[V / 2+i(i-1) / 2] /(V+1)$ for i from 1 to $\mathrm{V}$.

\section{References}

Bergemann, D., Morris S. [2013] Robust predictions in games with incomplete information, Econometrica 81, 1251-1308.

Bernheim, D.B. [1984] Rationalizable strategic behaviour, Econometrica 52, 1007-1028.

Che, Y., K., Gale I., L. [1998] Caps on political lobbying, The American Economic Review 88, 643651.

Dechenaux, E., Kovenock, D., Sheremeta, R.M. [2015] A survey of experimental research on contests, all-pay auctions and tournaments, Experimental Economics 18, 609-669.

Dekel, E., Jackson, M., O., Wolinsky A. [2008] Vote buying: general elections, Journal of Political Economy 116, 351-380.

Fudenberg, D., Tirole, J.[1986] A theory of exit in duopoly, Econometrica 54, 943-960.

Gneezy, U., Smorodinsky, R. [2006] All-pay auctions- an experimental study, Journal of Economic Behavior and Organization 61, 255-275.

Hendricks, K., Weiss, A., Wilson C. [1988] The war of attrition in continuous time with complete information, International Economic Review 29, 663-680.

Hörisch, H., Kirchkamp, O. [2010] Less fighting than expected, experiments with wars of attrition and all-pay auctions, Public Choice 144, 347-367.

Kosfeld, M., Droste, E., Voorneveld, M. [2002] A myopic adjustment process leading to best-reply matching, Games and Economic Behaviour 40, 270-298.

Lugovskyy, V., Puzzello, D., Tucker, S. [2010] An experimental investigation of overdissipation in the all-pay auction, European Economic Review 54, 974-997.

Pastine, I., Pastine, T. [2012] Incumbency advantage and political campaign spending limits, Journal of Public Economics 96, 20-32.

Pearce, D. [1984] Rationalizable strategic behaviour and the problem of perfection, Econometrica 52, 1029-1050.

Shubik, M. [1971] The dollar auction game: a paradox in noncooperative behaviour and escalation, The Journal of Conflict Resolution 15, 109-111.

Umbhauer, G. [2016] Game theory and exercises, Routledge Advanced Texts in Economics and Finance, Routledge Editors, London.

Umbhauer, G. [2017] Equilibria in discrete and continuous second-price all-pay auctions, convergence or yoyo phenomena, BETA Working Paper 2017-14, University of Strasbourg, France. 